\title{
S Doradus variables in the Galaxy and the Magellanic Clouds`
}

\author{
A. M. van Genderen \\ Leiden Observatory, Postbus 9513, 2300 RA Leiden, The Netherlands \\ e-mail: genderen@strw.leidenuniv.nl
}

Received 6 June 2000 / Accepted 10 October 2000

\begin{abstract}
The goal in writing this paper is five fold: (1) to summarize the scientific achievements in the 20th century on S Dor variables (or LBVs); (2) to present an inventory of these variables in the Galaxy and the Magellanic Clouds with a description of their physical state and instability properties; (3) to emphasize the photometric achievements of the various types of instabilities. Generally this seems to be a neglected item resulting in a number of misunderstandings continuously wandering through literature; (4) to investigate the structure of the S Dor-area on the HR-diagram; (5) to estimate the total numbers of S Dor variables in the three stellar systems. The position of the strong active $\mathrm{S}$ Dor variables in minimum brightness obey the following linear relation on the HR-diagram: $\log L / L_{\odot}=1.37 \log T_{\text {eff }}-0.03$. The relatively small dispersion of less active and supposed exand dormant S Dor variables with respect to this relation is twice as large at the blue side than at the red side. This might be caused by evolution to the WR stage and/or to high rotation. S Dor variables can be subject to five types of instabilities: the very rare genuine eruptive episodes (the "SD-eruptions"), two different brightening phases caused by slow pulsations (the "SD-phases"): one on a time scale of years, the other on a time scale of decades at a more or less constant luminosity and two types of microvariations: one on a time scale of weeks, the other on a time scale of about $100 \mathrm{~d}$. So far, no periodicities of light curve characteristics of any of these instabilities have ever been found. The durations of active and non-active stages are estimated for about half of the sample based on scattered magnitude estimations such as from historical records, and on modern monitoring campaigns. It would be a misunderstanding to believe that all S Dor variables should be always spectacular. It is estimated that most of them will not be spectacular at all for at least $70 \%$ of their lifetime as an S Dor variable.
\end{abstract}

Key words. catalogue - stars: variables - stars: supergiants

\section{A review of the scientific achievements}

\subsection{Introduction}

Now, at the beginning of the 21st century it is appropriate to summarize the scientific achievements in the field of S Dor variables (or LBVs: Luminous Blue Variables) and to make an inventory of them in the Galaxy and the Magellanic Clouds.

When Humphreys \& Davidson (1994) wrote their review paper on S Dor variables entitled: "Astrophysical Geysers-The Luminous Blue Variables", the Galaxy numbered 5, the LMC 6 and the SMC 1 "confirmed" of these variables. The list of "candidates" numbered 4, 4 and 0 stars, respectively. A few years later, Parker (1997) listed 6 new candidates for the Galaxy and 2 confirmed S Dor

* Tables 1 to 6 and 8 to 17 are only available in electronic form at http://www.edpsciences.org, Table 7 is only available at the CDS via anonymous ftp to cdsarc.u-strasbg.fr (130.79.128.5) or via

http://cdsweb.u-strasbg.fr/cgi-bin/qcat?J/A+A/366/508 Figures 2-10, 12, 14, 15, 17-19 are only available in electronic form at http://www.edpsciences.org, see Note added in proof variables and 1 candidate for the LMC. The list presented by Bohannan (1997) is very similar.

Apart from a concise description on the various aspects of S Dor variables (as I will call them throughout this paper, see Sect. 1.2), a list is presented of 34 confirmed members (including 8 so-called ex-/dormant members, see Sect. 1.2), 12 candidate members and 3 former candidates. Of the 46 objects (leaving out the 3 non-members) 21 belong to the Galaxy, 4 to the SMC and 21 to the LMC. They have been divided into four categories (defined in Sect. 2.1). For each category three tables are given listing a number of photometric and physical parameters, the reddening and the distance (all have been selected and/or have been made as homogeneous as possible), time scales and light amplitudes of the various types of instabilities, and whether ejecta are present. Each entry is accompanied by non-exhaustive reference numbers decoded in a separate table. It is not professed to be complete, after all, other references can be found in the quoted ones.

Further, for 18 objects a schematic light curve is presented, giving an impression of the past and present light variations. A few brightness estimates of LMC objects made in the 19th century are partly based on a compilation by Thackeray (1974) and partly on other 
sources such as the HD and HDE catalogues (the last one dated 1925). The ptm (photometric) and ptg (photographic) magnitudes of the HD catalogue are, depending on the declination, based on visual magnitudes of the Bonner Durchmusterung dated around 1850, of the Cordoba Durchmusterung and of the Cape Photographic Durchmusterung (both dated at the end of the 19th century) by applying a colour index correction if necessary (see the introduction to the HD catalogue).

A short discussion is devoted to a possible evolutionary connection between S Dor variables and the variable yellow hypergiants and some attention is paid to the present status of $\eta$ Car.

The structure of the S Dor (SD)-area in the theoretical HR-diagram is analyzed for the four categories mentioned above.

Finally, a discussion is presented on the total numbers of estimated S Dor variables in the three stellar systems.

\subsection{Nomenclature and general characteristics}

S Dor variables are a separate class of massive and very evolved stars. They are subject to a number of instabilities, often at the same time. Their origin is still largely a mystery, although there are a number of presumptions based on theories and models. Three types of photometrically observable instabilities belong to the "SDphenomenon", two others to the microvariations. One of the latters is typical for all variable super- and hypergiants: the $\alpha$ Cyg variables (thus including the $\mathrm{S}$ Dor variables, see Sect. 1.6).

I prefer to call them "S Dor variables" and not "LBVs", because this is the traditional designation given by Kukarkin et al. (1974), (to be more specific: "hot S Dor variables", since it is not excluded that also cooler supergiants can be subject to similar type of instabilities, see Sect. 3.2.5; de Jager \& van Genderen 1989; van Genderen 1991a; Stahl et al. 1990). In this way confusing names like "yellow LBVs" can be avoided.

An even more important reason not to call them LBVs is that all evolved massive stars are (micro-)variables, thus, also the blue ones, but only a very small fraction belongs to the S Dor variables.

The preference to keep the original name memorized, has been brought into practice by introducing the concept "S Dor (SD)-phase" for the cyclic phases of brightening on a time scale of years to decades and light amplitudes up to a maximum of $\lesssim 2^{\mathrm{m}} 5$ (van Genderen et al. 1997a,b; Sect. 3.4 of the present paper).

It should be avoided to call these cyclic SD-phases "eruptions" or "outbursts", because they suggest a period with violent mass ejections, which, as we know nowadays, is not the case (Sect. 1.5). The main cause of SD-phases are stellar radius and temperature variations. Sometimes, but not always, they are accompanied by a denser wind (Sects. 1.5 and 3.2.1). The few cases which showed genuine eruptions, called "SD-eruptions" and witnessed the last few centuries (Sect. 1.4), are P Cyg (17th century), $\eta$ Car (19th century), perhaps HD 5980 (1993-1995) in the SMC and a few in other galaxies (Humphreys 1999).

Humphreys (1999) suggested to name the just mentioned objects " $\eta$ Car-like variables", not only because of the eruptions, but also because of an excess luminosity during the eruption (see for $\eta$ Car: van Genderen \& Thé 1984; for P Cyg: Lamers \& de Groot 1992, de Groot \& Lamers 1992; for V12 in NGC 2403 and for SN 1961V: Humphreys \& Davidson 1994). After these eruptive periods, the four variables showed a post-maximum plateau, a second, lesser eruption and subsequently a strong obscuration by circumstellar dust (Humphreys et al. 2000). That these eruptive periods are fundamentally different from the SD-phases, which in the case of $\eta$ Car are visible as small-amplitude light oscillations (several $0^{\mathrm{m}} 1$ ) on a time scale of a few years, became obvious from a photometric analysis in 1984 by van Genderen \& Thé (1984: Sects. 9.2 and 9.6), see also Whitelock et al. (1983) and van Genderen et al. (1999: Sect. 4.1).

Since many S Dor variables seem to suffer from such eruptive periods at least once in their life time (Sect. 1.8), I believe that a separate name for stars like $\eta$ Car, P Cyg, etc., is not yet necessary, unless their light curves are really unique, i.e. when it will be shown in due course that other S Dor variables exhibit eruptive light curves different from those just mentioned. The eruptive light curve of the candidate S Dor variable HD 5980 in the SMC (in 1992 and 1993) looks quite different indeed (see for references Tables 11,12 and 13). That e.g. the variable star hidden in $\eta$ Car behaves as a normal S Dor variable also, is demonstrated by the $26 \mathrm{yr}$ (1974-2000) of multi-colour photometry undertaken by our group. It shows typical SD-phases with at times superimposed microvariations, although, often disturbed by a variable non-stellar light source (with especially in the near-UV peculiar periodicities). This light source could perhaps be a luminous accretion disk/hot spot system: see Sects. 3.2.2 and 3.2.6 and Tables 4, 5 and 6; van Genderen et al. 1994, 1995, 1999; 2001; de Groot et al. 1997a; Sterken et al. 1996b, 1999a,b).

\subsection{Criteria for $S$ Dor-membership}

The criteria, or signatures for SD-membership are extensively discussed by Humphreys \& Davidson (1994). Supplemented with new insights, they can be summarized as follows:

a) visible ejecta (although only $40 \%$ of the present compilation has visible ejecta, Sect. 1.8), likely caused by (an) SD-eruption(s);

b) spectroscopic (variable) characteristics indicating a high luminosity, a high mass loss rate $\left(\sim 10^{-5} M_{\odot} \mathrm{yr}^{-1}\right)$, an extended atmosphere and ejecta containing $\mathrm{CN}$ or $\mathrm{CNO}$ processed material. This type of chemistry as well as the dust composition, is consistent with a RSG (red supergiant) origin (Sect. 1.7); 
c) a photometric variability of up to 2.5 on a time scale of years to decades and even to centuries, the socalled SD-phases (Sects. 1.5 and 3.4). One of the typical characteristics is that the light variations show a large variety. The colour variations are crucial for the detection of the SD-phases and therefore a very strong diagnostic tool: due to the excursions on the HR-diagram at a more or less constant luminosity, the colour indices become red when the star (if hotter than $\sim 7000 \mathrm{~K}$ ) brightens and blue when the star becomes fainter. This habit appears to be easily detectable, even if the light ranges of SD-phases are as small as 0.1 or even smaller, thus, they should not be confused with the microvariations which have much shorter time scales (Sect. 1.6). In this way a number of candidates could be classified as genuine $\mathrm{S}$ Dor variables.

Spectroscopically suspected S Dor variables turned out to fulfil without any exception the photometric criteria, if enough multi-colour photometric observations were made. Therefore, if spectroscopic observations, as well as a proof for ejecta are (still) lacking, I applied the reverse reasoning to identify S Dor variables only based on the photometric multi-colour behaviour.

Ofpe/WN9 stars will not be considered as suspected or candidate S Dor variables (such as S 9), unless they show an N-enriched spectrum and stellar ejecta such like S 61 (= Sk-69 266, see Tables 8, 9 and 10). To cite Bohannan (1997): “... Ofpe/WN9 stars have been proposed as quiescent LBVs. As appealing as that is, this call cannot be made on spectroscopic features alone". Therefore, the stars studied spectroscopically in the UV by Smith Neubig \& Bruhweiler (1999), although showing SD-characteristics in the UV, are not included in the present list of candidates, unless they were already identified as genuine S Dor variables by other criteria.

\subsection{The $S$ Dor (SD)-eruptions}

The extremely powerful eruptions of a small number of $\mathrm{S}$ Dor variables mentioned in Sect. 1.2 released a total energy of $10^{47.3}-10^{49.5}$ ergs (Humphreys et al. 2000). There are different theoretical approaches to these SDeruptions, although, to cite Davidson \& Humphreys (1997): "Instabilities of this type are so complex that it is difficult to be sure that competing models are fundamentally different". However, all authors agree that the $L / M$ ratio is likely higher than for other stars at the same location on the HR-diagram, thus, they must have lost a lot of mass already. Humphreys \& Davidson (1994) discussed all views and models giving the state of affairs up to 1994 . They believe that the sub-photospheric instability models are most promising (outer layers below $210^{5} \mathrm{~K}$ are dynamically isolated from the stellar interior because of the high opacity due to iron, and can easily become unstable). This is the hypothetical "modified Eddington limit", in which "modified" indicates that the opacity is temperature- and density-dependent. (The classical Eddington limit is an upper limit to the ratio $L / M$ for a nearly static stellar atmosphere).

Since then a number of other models and mechanisms were proposed:

Langer et al. (1994) discussed a new scenario for the evolution of massive stars $\left(M_{i} \gtrsim 40 M_{\odot}\right)$ by introducing mass-loss in addition to the radiation-pressure driven wind, resulting in better agreement with the observations than previous scenarios.

Cox et al. (1997) gave a summary of the mechanisms proposed to destabilize S Dor-envelopes up to 1996.

Subsequently, Stothers \& Chin (1997) interpreted e.g. $\eta$ Car as a star burning hydrogen in its core, while repeatedly encountering ionization-induced dynamical instability, with a time scale of $3-6 y r$ on the average. This time scale for violent cycles agrees remarkable well with the observed frequency of $\sim 1^{\mathrm{m}}$-peaks superimposed on the eruptive light maximum between 1827 and 1857. Note that the light curve (Fig. 11) shows a few gaps during this time interval of as long as $\sim 5 \mathrm{yr}$, so that more peaks may have occurred. Then, two more peaks appeared: $13 \mathrm{yr}$ later in 1870, and again $20 \mathrm{yr}$ later in 1890. Also the other cases discussed by Humphreys et al. (2000), showed eruptive episodes characterized by a set of individual eruptions with a sharply declining frequency.

Owocki \& Galay (1997) suggested that when an evolving star responds to a super-Eddington condition, it will develop a convective outer layer which will be blown up mimicking a RSG. This envelope may then become detached, due to a density inversion, so that an S Dor variable is born.

A quite different possibility has been proposed by Sterken et al. (1997) based on the high noise, extending over a wide frequency range exhibited by the light variations of the ex- or dormant S Dor variable $\zeta^{1}$ Sco. The presence of this noise, unpredictable, but to some degree in step with the microvariations, could from time to time amplify the regular long-term oscillations beyond expectation leading to an eruption. Such amplification of weak signals by associated noise, known as stochastic resonance, could lead to unexpected triggerings when combined with long-period oscillations, especially when the star is in a state of dynamical instability.

In the proceedings of the IAU Coll. 169 held in Heidelberg in 1998, a number of papers were devoted to the instabilities in S Dor envelopes, in particular to the SD-eruptions (e.g. Guzik et al. 1999; Ødegaard 1999) and all types of pulsations (the latter might play a role in the observed microvariations, see Sect. 1.6).

During the workshop on $\eta$ Car, held in Montana 1998, special emphasis was given to its SD-eruption and the creation of the bipolar nebula. The model of Cassinelli (1999) includes binarity and that of Iben (1999) even a tertiary companion. These models describe with straightforward physics SD-eruptions leading to a bipolar nebula. However, other S Dor variables excist with bipolar nebulae without being a binary as far as we know. 
With hydrodynamical models of the evolution of bipolar ejecta, in particular that of $\eta$ Car, Langer et al. (1999) find support for the general conjecture that SDeruptions occur when the stars approach the Eddington limit. Their models imply that initial stellar rotation rate and angular momentum have influence on the occurrence of SD-eruptions and that they are very important to the evolution of very massive stars.

Stothers' (1999a) calculations show that rotation influences the hydrostatic structure as well, the course of the evolution and in an indirect way the star's $L / M$ ratio and consequently the onset of dynamical instability, an appealing mechanism discovered by Stothers \& Chin (1993, 1994, 1995, 1996).

All these models do not consider any possible influence of the frequently occurring SD-phases (which are in fact slow pulsations, Sect. 1.5) and the microvariations (which are always present, Sect. 1.6) on the eruptive instabilities and on the production of ejecta. An exception so far with respect to $\eta$ Car, is the study of Stothers (2000). He examines the effect of the enormous stellar wind on the envelope structure, assuming that $\eta$ Car possesses a very massive hot main-sequence star, only slightly evolved. The high mass loss must dynamically perturb its outer envelope down to the two main iron convection zones of which the turbulent energy can be directly transformed into mass ejections. Besides, Stothers (2000) found that secular oscillations of the outer envelope, caused by the runaway mass loss, is potentially able to account not only for the cycles of visual brightenings (the SD-phases, Sect. 1.5), but also for the 1827-1857 eruptive period.

Whatever the truth is about the SD-eruptions, they seem to occur rarely in view of the low number of ejecta per S Dor variable. There are even S Dor variables without any ejecta (Sect. 1.8).

\subsection{The $S$ Dor (SD)-phases}

The SD-phases (see also Sect. 1.3, c) are presumably largely phases of variable radius and temperature. This was first suspected by van Genderen (1982), and later confirmed by the study of Leitherer et al. (1989), while de Koter et al. (1996) showed that a reduced effective gravity should take part in the process. The luminosity stays more or less constant. One of the exceptions is S Dor; here the change in $M_{\text {bol }}$ is close to $1^{\mathrm{m}}$ (van Genderen et al. 1997a). An SD-phase is apparently a kind of pulsation, especially by the envelope (see also Lamers et al. 1998: Sect. 5.3). It appears that there are two types: one on a time scale of years and another one on a time scale of decades (Sect. 1.5.1).

The physical implications, amongst others with respect to the mass loss rate (see below) are still not understood (Schmutz 1997). His suggestion is that an SD-phase "is a sort of small giant eruption that never makes it to infinity". Most likely we see the stellar photosphere all the time from minimum to maximum light (and not an expanding optically thick pseudo-photosphere as thought formerly, however, see Sect. 3.2.1).

It is of interest to note that the S Dor-phases of $\eta$ Car seem to be more or less sensitive for tidal forcing of a supposed companion with an excentric orbital revolution of $5.5 \mathrm{yr}$ (van Genderen et al. 2001): around the periastron passages the light is in, or close to a maximum. Such a possibility has been suggested by Stothers \& Chin (2000).

It is doubtful whether the physical process of the SDphases can be compared with a terrestrial geyser. This geyser model has been introduced by Maeder $(1989,1992)$. He elaborated the density inversion below the photospheres of cool supergiants (temperature below $9000 \mathrm{~K}$ ), discovered by Böhm-Vitense (1958), a probable cause of eruptive episodes. Although the S Dor variables discussed here are hotter, Humphreys \& Davidson (1994) suggested that their characteristics allow the comparison with geysers. The duration of the quiescent period should be correlated with the size of the preceding eruption. However, the investigation of such a type of correlation for AG Car did not support that (van Genderen et al. 1997a). What we did find is that the durations of the SD-phases (on a time scale of years) appear to be linearly proportional to the visual light amplitude. The geyser model may be physically related to the dynamical unstable yellow/red SD-stage, as follows from the calculations of Stothers \& Chin (1996) (see also Sect. 1.8 and the comments by Humphreys \& Davidson 1994).

Contrary to former beliefs, a positive correlation between the brightness variation due to the SD-phases and the mass loss rate appeared to be not always true (Leitherer et al. 1989, 1994; de Koter et al. 1996). Whether $\dot{M}$ increases or decreases during an SD-phase depends on the temperature change, the proximity to the Atmospheric Eddington Limit (AEL, Lamers 1997), and the bistability jumps (Pauldrach \& Puls 1990; Lamers 1997).

\subsubsection{Two types of SD-phases}

The study of a century of photometric observations of AG Car and S Dor revealed the presence of two different SDphases. If both are present simultaneously, the shorter one, time scale of years: $t<10 \mathrm{yr}$, is clearly superimposed on the longer one, time scale of decades: $t \gtrsim 20 \mathrm{yr}$ (van Genderen et al. 1997a). The first one was called a normal (N)-SD phase, the second one a very long-term (VLT)-SD phase. So far, no SD-phases were found with cycle lengths between 10-20yr. After having studied so many S Dor variables in more detail, I want to introduce the more convenient names: short (S)-SD and long (L)-SD phase, respectively.

SD-phases are probably caused by two different instability regions. This suspicion is based on the fact that amplitude and shape of the S-SD phases are clearly modulated by the ongoing L-SD phase (van Genderen et al. 1997a; Sterken et al. 1996a). So far, there is no theoretical support for this assumption. In the models of 
Stothers \& Chin (e.g. 1995) the "secular cycles" can perhaps be identified as the SD-phases, but their models do not predict a sharp physical (or even temporal) division between S- and L-SD phases. The actual length of a particular "secular cycle" depends on the dynamical mass-loss rate. However, the observed S-SD cycle durations of the order of a year are too short to be attributed to the secular cycles (Stothers, priv. comm.).

It is also possible that a period-modulation excists, i.e. that the L-SD phase influences the arrival times of the light maxima of the S-SD phases (Sects. 1.5.2 and 1.5.3). It appears that most of the $\mathrm{S}$ Dor variables are subject to both types of SD-phases, albeit not always simultaneously (see light curves presented by van Genderen et al. 1997b; van Genderen \& Sterken 1996; Sterken et al. 1998).

\subsubsection{Periodicities/cyclicities of $S$ Dor variables}

Important for the interpretation and modelling of the SDphases is the fact that multi-periodicities/-cyclicities have been found in the S-SD phases of a number of S Dor variables (van Genderen et al. 1997a,b; Sterken et al. 1996b, 1997a, 1998). AG Car shows a very pronounced primary periodicity of $371.4 \mathrm{~d}$ with beat cycles (Sect. 1.5.3).

So far, no theoretical models predict two different types of SD-phases (Sect. 1.5.1), nor any (multi-) periodicity (e.g. Maeder 1989, 1992, 1997; Glatzel \& Kiriakidis 1993; Cox et al. 1997, 1999; Guzik et al. 1997, 1999; Glatzel 1997). Stothers \& Chin's $(1995,1996)$ models reveal a mechanism for dynamical instabilities. From their evolutionary models, "periods" were predicted of the overall "secular cycles" of $\eta$ Car, AG Car and S Dor (i.e. the cycles from hot to cool portions of the dynamical unstable stage and vice versa), which agree with the observed ones (S-SD phases) to within a factor of two.

So far, no repetition of light curve characteristics has ever been found for the SD-phases. It seems that Dorfi \& Feuchtlinger's (1998, priv. comm. to Guzik et al. 1999) models of S Dor-type envelopes exhibit regular pulsations.

\subsubsection{The multi-periodic character of AG Car}

The primary period of the S-SD phases of AG Car appeared to have been stable during a century: $P_{0}=$ $371.4 \mathrm{~d} \pm 0.6 \mathrm{~d}$ (m.e. $=$ mean error). The oscillating $\mathrm{O}-\mathrm{C}$ (= observed epochs of maximum light minus the computed epochs of maximum light) values suggested the presence of a first beat period $P_{\mathrm{b} 1}=21.6 \mathrm{yr}$ and a possible second beat period $P_{\mathrm{b} 2}=4.7 \mathrm{yr}$ (van Genderen et al. 1997a). New epochs of maximum light in 1995 and 1996 supported these results (Sterken et al. 1996a).

The last and most reliable part of the $21.6 \mathrm{yr}$ beat cycle (represented by the $\mathrm{O}-\mathrm{C}$ diagram, see below) appears to have a similar shape as the L-SD cycle. I believe that this is suspect. Therefore, it is possible that $P_{\mathrm{b} 1}$ has been caused by the modulation of $P_{0}$ by the L-SD

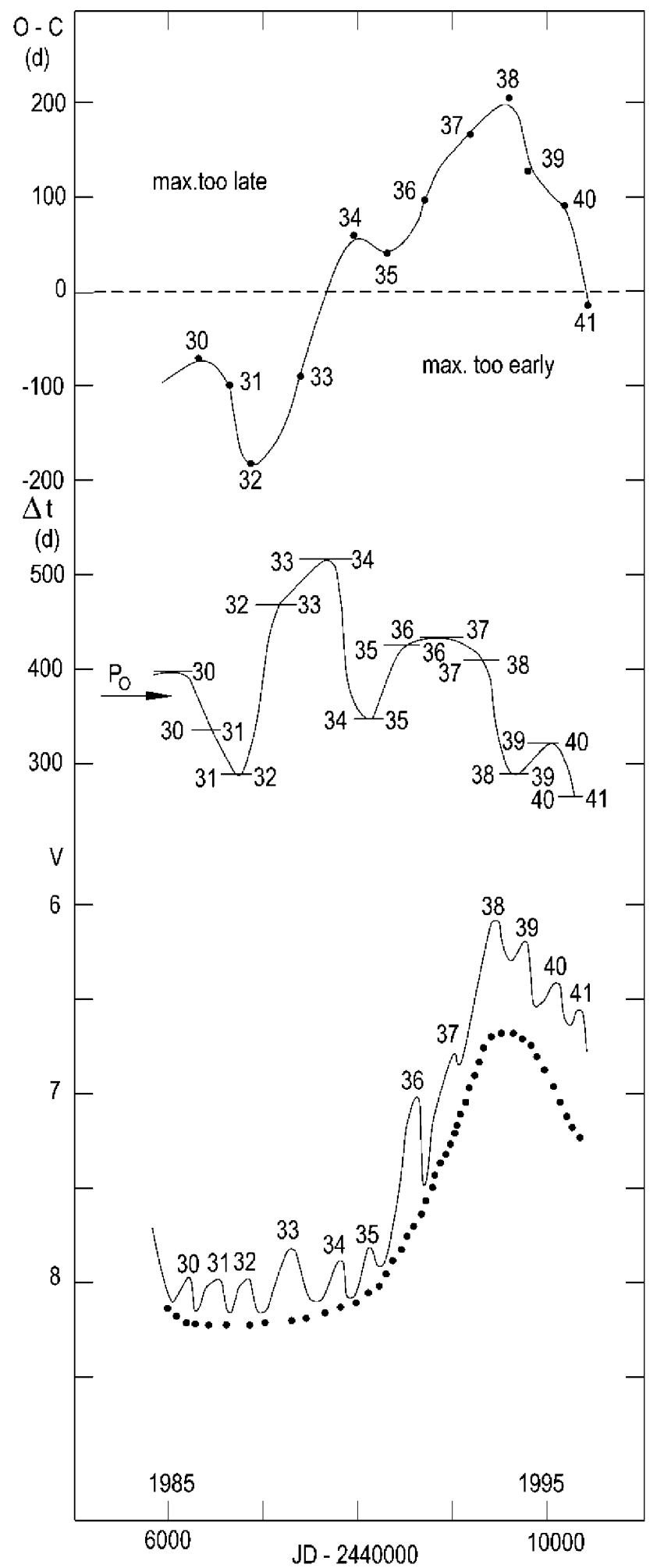

Fig. 1. From top to bottom: the (O-C)-, $\triangle t$ - and the schematic light curve of AG Car from maximum No. 30 onwards (full line; the dotted curve represents the underlying L-SD cycle)

cycle $(\sim 20 \mathrm{yr})$ instead of by a secondary period $P_{1}=$ $390 \mathrm{~d}$. This is explained in Fig. 1, from top to bottom:

Panel 1: the O-C curve for the S-SD phases (numbers from van Genderen et al. 1997a and Sterken et al. 1996a), 
based on the linear ephemeris with $P_{0}=371.4 \mathrm{~d}$ starting with maximum 30.

Panel 2: the trend of the duration $\triangle t$ (d) between two successive maxima. It illustrates the secondary oscillations of Panel 1 more clearly, representing the possible beat cycle $P_{\mathrm{b} 2}=4.7 \mathrm{yr}$. Each horizontal line piece, marked by the numbers of the two maxima has a length $\triangle t(\mathrm{~d})$. The estimated error amounts to $\pm 35 \mathrm{~d}$. It appears that $\triangle t$ hovers between $200 \mathrm{~d}$ and $540 \mathrm{~d}$. Using the formula $1 / P_{\mathrm{b} 2}=\left|1 / P_{2}-1 / P_{0}\right|$ and depending on whether $P_{2}$ (its meaning is explained below) is shorter or longer than $P_{0}$, one finds $P_{2}=305 \mathrm{~d}$ and $475 \mathrm{~d}$, respectively.

Panel 3: the schematic light curve from maximum 30 onwards. The dotted curve, touching most of the minima represents the shape of the underlying L-SD cycle on which the S-SD phases are superimposed.

The rising trend in the $\mathrm{O}-\mathrm{C}$ values (Panel 1) between e.g. maxima 34 and 38 means that the maxima arrive progressively later due to the accumulation of too long S-SD cycles, thus, too large $\triangle t$ values compared to the average $\triangle t\left(=P_{0}\right)$. The opposite appears for the subsequent steep decline after maximum 38. That the $\mathrm{O}-\mathrm{C}$ curve in panel 1 and the dotted L-SD curve in panel 3 look similar is evident. This may not be accidental, and due to a modulation effect by the L-SD cycle on the periods $P_{0}$ and $P_{2}$. If this should be correct, the beat $P_{\mathrm{b} 1}=21.6 \mathrm{yr}$ has nothing to do with the interference of $P_{0}$ with a secondary period $P_{1}$ (= $390 \mathrm{~d})$. The latter is then obsolete, and $P_{2}(305 \mathrm{~d}$ or 475 d) should be called $P_{1}$.

The ratio between these two, $P_{0}(=371.4 \mathrm{~d})$ and $P_{1}(=$ $305 \mathrm{~d}$ or $475 \mathrm{~d}$ ), amounts to approximately $4 / 5$ (if $305 \mathrm{~d}$ is used), or $5 / 4$ (if $475 \mathrm{~d}$ is used), a ratio which is often found among multi-periodic pulsating stars.

\subsection{S Dor variables as a subgroup of $\alpha$ Cyg variables. The two types of microvariations}

All evolved massive stars, generally referred to as $\alpha$ Cyg variables, including the $\mathrm{S}$ Dor variables, show currently a photometric microvariability with light amplitudes $\lesssim 0$ m. 2 . Note that the light amplitude of the microvariations can be larger than that of the S-SD phase (e.g. HD $34664=$ $\mathrm{S} 22$ in Fig. 16). The time scales, often called semi- or quasi-periods, are of the order of days (the hot members) to months (the cool members) (van Genderen 1989, 1991a; Sterken 1989; van Leeuwen et al. 1998; van Genderen \& Sterken 1999 and references therein).

Although the precise evolutionary connections and the physical differences between $\alpha \mathrm{Cyg}$ variables and S Dor variables are still unclear, I tentatively considered the last ones as a small sub-group of the first ones (van Genderen et al. 1988). The photometric behaviour of the famous S Dor variable P Cyg seems to support this view. According to the models the star should be dynamically stable at present (thus no SD-phases, but see Note added in proof; Sect. 3.2.2), but it does show microvariations. It also appears that in the same temperature domain S Dor variables and normal $\alpha$ Cyg variables, show almost the same type of microvariations. Often, the microvariations of S Dor variables near minimum light show fewer secondary features, and the amplitudes (or the "maximum light amplitudes": MLA) appear to be somewhat larger (van Genderen 1989, 1991a; van Genderen \& Sterken 1996). Many S Dor variables near minimum light might still show very weak SD-phases which contribute to the MLA's.

Analyzing the S Dor variables with a strong SDactivity, we noted a peculiar phenomenon. At or near minimum light they show $\alpha$ Cyg-type microvariations with quasi-periods of the order of days to weeks (like normal $\alpha$ Cyg variables). As soon as they pass a certain temperature domain on their way to maximum light, somewhere between $20000 \mathrm{~K}$ and $10000 \mathrm{~K}$, another type of microvariation emerges rather abruply (within a few months), with a much longer time scale: the " $100 \mathrm{~d}$-type" variation. That is to say, the time scale is often of the order of $100 \mathrm{~d}$, but can range from $50 \mathrm{~d}$ to $150 \mathrm{~d}$, of the same order as normal $\alpha$ Cyg variables (non-S Dor variables) of the same temperature. The amplitudes are still $\lesssim 0.2$ (see references above). However, the colour behaviour of the $100 \mathrm{~d}$-type variations is often red in the light maxima instead of blue as for the shorter microvariations mentioned above (e.g. R 40 in van Genderen et al. 1997b: Figs. 8-10) and often more chaotic (e.g. S Dor and R 127 in van Genderen et al. 1997a and 1997b, respectively).

I speculate that they could correspond to the oscillations found by Stothers \& Chin $(1995,1996)$ in their models and called "relaxation oscillations" (a kind of pulsation superimposed on a dynamical unstable structure) due to the kappa-mechanism. They occur during the cool part of the "secular cycles" in the models as well as in the observed maxima of the SD-phases. The predicted periods are also in the order of months! Stothers' (1999c) idealized hydrodynamical models reveal very small bolometric light amplitudes, less than 0.2 , which is likely to be generally true, even for realistic models.

We found three S Dor variables where both types of microvariations were seen together: the short one superimposed on the long one. The first two are HR Car and R 85 (Fig. 13), but only for a few months (van Genderen et al. 1990, 1997b, 1998b). The third is P Cyg, but here both oscillations, $18.3 \mathrm{~d}$ and $100 \mathrm{~d}$-type, are already seen together for many decades up to the present day (de Groot et al. 2001). Obviously, the physical state of P Cyg is very close to the switching point for a long time. Apart from these two types of microvariations, P Cyg also shows a very low amplitude oscillation with a cycle length of $\sim 3000 \mathrm{~d}$ (de Groot et al. 2001), which is presumably an S-SD phase (see Table 6), and an apparent brightness rise due to evolution to the red (Lamers \& de Groot 1992; de Groot \& Lamers 1992). Thus, in more than one respect $\mathrm{P}$ Cyg is an exceptional case (see also Sects. 1.7 and 3.2.2).

Apart from the enigmatic way in which the two types of microvariations succeed each other, the 100 d-type ones 
offer another riddle: it is quite peculiar that the time scales of e.g. R 127 and HR Car, hardly changed during the brightness rise of $\sim 1^{\mathrm{m}}$ in $V_{\mathrm{J}}$ (van Genderen et al. $1997 \mathrm{~b}$ : Sect. 4.2). This creates a paradox if one wants to explain them as a result of stellar radial pulsations, non-radial pulsations offer an acceptable alternative. But how to explain the (temporarily) simultaneous excitation of both microvariations? So far, theoretical studies do not predict such a phenomenon. The switch from one to the other could be explained by physical changes, such as the density structure during expansion and contraction. However, this cannot offer an explanation for the simultaneous presence of both types of microvaraitions.

There are different views on the cause and the physical background of microvariations. Amongst others, they were linked with the "strange-mode" instabilities (oscillation modes recovered in linear, non-adiabatic calculations for stars with high $L / M$ ratios), see e.g. Kiriakidis et al. (1993, 1997), Soukup et al. (1994) and Cox et al. (1995). See also the reviews by Gautschy \& Saio (1995, 1996).

Sterken (1989) was the first to suggest that the SDdomain on the HR-diagram is an extension of the instability domains of the non-radial $(l>0)$ pulsators in the gmode: $\beta$ Cep variables and SPBs (slowly-pulsating B-type stars) (see also Waelkens et al. 1998; Lamers et al. 1998). This seems to be supported by theoretical calculations (Pamyatnykh 1998) which also indicate that the above mentioned instability domain covers a much wider region than predicted for the strange-modes. Further, Lamers et al. (1998) found that the observed periods of the microvariations (often near minimum brightness) are much longer than those caused by the strange modes. According to Gäng's (2000) analysis of spectroscopic time series of HD 160529, radial and non-radial pulsations are probably present.

\subsection{The evolutionary status}

Most of the variable supergiants can be found on the blue side of the HR-diagram. This agrees with the various theoretical models (e.g. Lovey et al. 1984; Stothers \& Chin 1994, 1995, 1996; Schaller et al. 1992; Langer et al. 1994; Vanbeveren et al. 1998). The excess of blue supergiants is possibly due to the widening of the main sequence and their longer lifetimes.

$S$ Dor variables are mainly concentrated in the blue part of the HR-diagram as well. According to Stothers \& Chin $(1994,1995,1996)$ this is due to their much longer lifetime in their second (blue) phase of dynamical instability $\left(\sim 10^{4} \mathrm{yr}\right)$ than in their first as a yellow supergiant $\left(\sim 10^{3} \mathrm{yr}\right)$. Supergiants on a blueward track, which are a small minority due to their faster evolution, should show processed material at their surface like presumably the $\alpha$ Cyg variable HD 157038, a B1/2 IaNtype star (Lennon \& Dufton 1986, see for its photometric variability van Genderen et al. 1989).
Indeed, most of the S Dor variables show He- and N-enriched and O-poor circumstellar ejecta, pointing to CNO-processed material (e.g. L. Smith 1997). Also the dust composition of a number of S Dor variables (e.g. AG Car, WRA 751, R 71) points to an evolutionary connection with RSG (e.g. Viotti et al. 1988; Robberto et al. 1993; L. Smith et al. 1997, 1998; Voors 1999), although evolutionary tracks of e.g. Schaller et al. (1992) of stars with $M_{i} \gtrsim 40 M_{\odot}$ do not reach the RSG region at all, unless one introduces a mass loss in addition to the radiation pressure driven wind like Langer et al. (1994) did. Further support for a possible RSG-connection comes from the hydrodynamical models of the nebulae of e.g. P Cyg and AG Car by Garcia-Segura et al. (1996a,b), which imply that these stars evolved through what they call: a "blueward excursion". Yet, P Cyg is the only S Dor variable which lacks any dust in its ejecta (Voors 1999). (It is however not clear whether they also mean an excursion starting right from the RSG domain. Their evolutionary track for a star with an initial mass of $60 M_{\odot}$, does show a returning point in the RSG domain).

Initially, there was a discrepancy with regard to the $\mathrm{H}$-content in mass $(X)$ at the surfaces of $\mathrm{S}$ Dor variables amounting to $\sim 0.36$ (see the compilation by Stothers 1999b), while post-RSG should have 0.10-0.20 (Maeder 1997 ), or $\sim 0.18$ (Stothers 1999b). However, this seems to have been solved now by Stothers \& Chin (2000). If one adopts the Schwarzschild (temperature-gradient) criterion for convection instead of the Ledoux (densitygradient) criterion, a fully convective (thus chemically homogeneous) zone develops just above the H-burning shell, with $X$ approximately equal to 0.35 . The star would then be in the second (blue) S Dor stage.

On the other side, Maeder (1999) concluded that due to stellar rotation, some He- and N-enrichments at the stellar surface already occur during the MS-stage for not too high rotational velocities, thus it is not quite abnormal to find slightly enriched supergiants. Further, the computations indicate that rotation makes the star enter the WR-stage during the MS-stage, thus preventing an S Dorand RSG-stage altogether.

Also with respect to the scheme of evolution of all massive stars, there is still no consensus: different sequences of stellar types exist (see e.g. Langer et al. 1994; Stothers \& Chin 1996; Vanbeveren et al. 1998).

\subsection{The number of stellar ejecta and their ages}

In Tables 1, 4, 8 and 11 it is indicated whether circumstellar gas and/or dust nebulae have been detected. It appears that only $40 \%$ of the S Dor variables, including 8 candidates in these statistics for which the SD-membership is likely, possess with certainty one or more visible ejecta. (There is no statistically significant difference between the percentages of the different categories of S Dor variables defined in Sect. 2.1). It is not excluded that all have a neutral-H nebula, thus not easily detectable. Such nebulae 
are relics from previous evolutionary stages, such as e.g. a pseudo-RSG/super-Eddington phase with gentle ejections, as postulated by L. Smith et al. (1998). The visible ring nebulae around $25 \%$ of the WR stars, generally supposed to be partly the descendants of S Dor variables, or at least closely related to them, may be caused by the interaction of the fast WR wind with the slower winds of the RSG- and SD-mass loss episodes (Marston et al. 1994; Marston 1999). (It has also been suggested that the most luminous WNL stars may evolve into S Dor variables, rather than vice versa, after which they enter a second WR stage (Langer et al. 1994; Walborn 1989). Such a different history has consequences for the chemical composition of the WR nebulae, Garcia-Segura et al. 1996a,b.)

The great homogeneity of the properties of the ejecta generally suggest an interacting wind scenario by nonisotropic outflows. Thus, they are created by an interaction of fast moving gas overtaking slow moving gas (e.g. Icke 1981; Garcia-Segura et al. 1996a,b; L. Smith 1997; Dwarkas \& Balick 1998; Langer et al. 1999).

The visible ejecta have dynamical ages between $10^{2} \mathrm{yr}$ and $710^{4}$ yr (e.g. Nota et al. 1995a; Nota \& Clampin 1997; Smith et al. 1998). According to the dynamical evolution computations of Garcia-Segura et al. (1996a,b) visible ejecta have a lifetime of $\sim 10^{4} \mathrm{yr}$, which is of the same order.

The masses of the visible ejecta are at most a few solar masses (e.g. Nota \& Clampin 1997), thus not sufficient to explain the current relatively low masses of the S Dor variables. According to Stothers \& Chin's calculations (1996) the present blue phase of instability is also too short: $10^{3}$ $10^{4} \mathrm{yr}$, for a substantial mass loss. Thus, the major mass losses should have occurred during the preceding dynamical instability stage in the yellow- or RSG-stage as well as by the normal stellar wind since the MS-stage. The intervening stage from the RSG- to the blue SD-stage is expected to last $\sim 610^{4} \mathrm{yr}$ (Stothers \& Chin 1996). In other words, most of the ejecta we observe today are presumably from the present blue SD-stage. Any ejecta formed in the yellow or red stage must have been dispersed into space.

As far as we know, most visible ejecta are single. There are a few exceptions: AG Car has two ejecta (Nota \& Clampin 1997) like R 127 (Appenzeller et al. 1987; L. Smith et al. 1998), HR Car has at least three ejecta (e.g. Voors 1999), P Cyg has four ejecta (Barlow et al. 1994; Meaburn et al. 1996; Skinner et al. 1998; see also the review on P Cyg by Israelian \& de Groot 1999). The differences in dynamical ages amount to from a few centuries to $510^{4}$ yr.

The largest conundrum of all: $\eta$ Car, with its bipolar Homunculus, $160 \mathrm{yr}$ old, and with N-rich fast moving knots and strings, must have been active on various occasions up to $1000 \mathrm{yr}$ ago (Walborn et al. 1978; Weis et al. 1999; Bohigas et al. 2000). A second, but older (a few $10^{3} \mathrm{yr}$ ) bipolar shell has been found by Bohigas et al. (2000), but its chemistry shows no trace of chemical processing. The Homunculus has an onion-like structure,

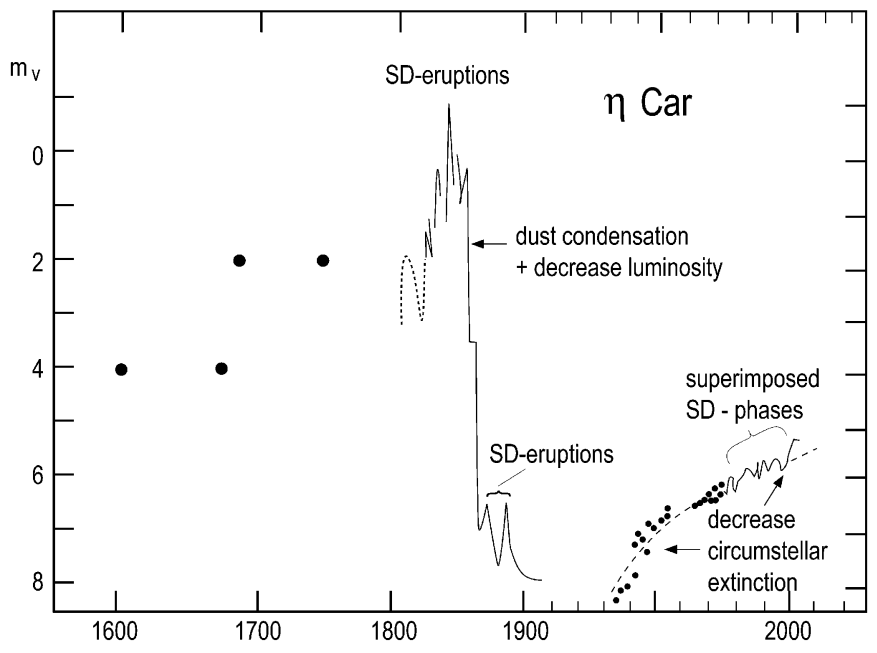

Fig. 11. The schematic light curve (ptm, ptg and $V_{\mathrm{J}}$ ) versus date of $\eta$ Car between 1600 and 2000 (last observations made in February 2000). Note that the horizontal scale after 1900 is twice as large as before 1900. Dashed curve: the "secular rise" due to the decrease of circumstellar extinction (mainly self-extinction of the Homunculus) according to the model of van Genderen et al. (1994, see also van Genderen et al. 1995, 2001). Dots are averages of time series and the oscillating curve represents a series of monitored SD-phases sketched on scale. See for further details the notes to Tables 4, 5 and 6

with sub-shells (Pantin \& Le Mignant 2000). The light curve (Fig. 11) also suggests the occurrence of a series of $\sim 1^{\mathrm{m}}$-eruptions between 1827 and 1857 with a repetition time $\lesssim 5$ yr. Apart from that there were also SD-eruptions around 1870 and 1890 (Sect. 1.4). However, it seems that their ejecta have another morphology (e.g. Davidson et al. 1997; N. Smith et al. 1998).

It can be concluded that at least $40 \%$ of S Dor variables, suffer a small number of eruptive episodes during their present (second) blue lifetime. The observed eruptive episodes appear to last a few decades and consist of a set of distinct eruptions. These eruptions are visible as $\sim 1^{\mathrm{m}}-3^{\mathrm{m}}$-peaks, in the beginning on top of an enhanced brightness. Subsequently the brightness drops, partly by circumstellar dust, and a few more eruptions occur (see light curves by Humphreys et al. 2000). The interval between such eruptive episodes may amount to a few centuries up to a few $10^{4} \mathrm{yr}$.

The overall impression is that $\mathrm{S}$ Dor variables lose most of their mass in previous evolutionary stages by the stellar wind and by eruptions caused by dynamical instabilities and not in their current lifetime as hot S Dor variables. The present visible ejecta are the relics of eruptive episodes from their present hot stage. S Dor variables which probably lack any ejecta, did not (yet) suffer from recent eruptions. 


\section{The inventory of the four categories of $S$ Dor variables}

\subsection{A sub-classification based on the strength of the SD-instability}

Lamers (1987) classified the individual SD-phases by means of the size of the light amplitude (note that they were called "eruptions" by him as well as by most researchers, which I advise against). In the present paper, the S Dor variables are classified according to their maximum light amplitude reached in the 20th century. Thus, this is a classification on a centennial basis. It is quite possible that a few will enter another category in the 21 st century. Some behaved differently in the 19th century. A reason to choose this kind of classification is to investigate whether the size of the instability has any influence on its present (at the turn of the 21st century) position on the theoretical HR-diagram.

Our knowledge of the photometric behaviour of S Dor variables is based on a strongly varying degree of coverage of the light curve. Variables like AG Car, S Dor, $\eta$ Car, HR Car and P Cyg have been photometrically monitored for almost the whole 20th century. For others, only the last few decades were covered by photometric observations, while e.g. the HD and HDE catalogues furnish brightness data for earlier dates (Sect. 1.1). Thus, the knowledge of the photometric behaviour in the first half of the 20th century is often only fragmentary, so that some caution is appropriate.

The centennial classification is defined as follows:

a) The strong-active (s-a) members: light amplitudes $>0{ }^{\mathrm{m}} 5$. The cycle lengths of the SD-phases are $<10 \mathrm{yr}$ and $\gtrsim 20 \mathrm{yr}$ (S-SD and L-SD phases, respectively). They are listed in Tables 1, 2 and 3;

b) The weak-active (w-a) members: light-amplitudes $<0$. 5 . The cycle lengths of the SD-phases are $<10 \mathrm{yr}$, thus they are S-SD phases. It is evident that the detection of low amplitude (say $<0.2$ ) variations on a time scale of decades (representing the L-SD phases) is difficult due to too short time bases of the observations. They are listed in Tables 4, 5 and 6;

c) The ex- and dormant (ex-/dormant) members. These objects showed no SD-type photometric variability in the 20th century as far as the scattered observations allow such a conclusion (though they do show the microvariations like all $\alpha$ Cyg variables, if photometrically well observed). Usually, they are, or have been suspected members on account of one or two of the other criteria listed in Sect. 1.3. For some specimens a strong photometric variability in the 19th century, or even earlier, has been taken as an important criterium for membership. If they would have been monitored photometrically more extensive, a few might in fact be w-a S Dor variables. They are listed in Tables 8, 9 and 10 ;

d) Candidate SD variables (coined such in the literature) and former candidates (candidates rejected in

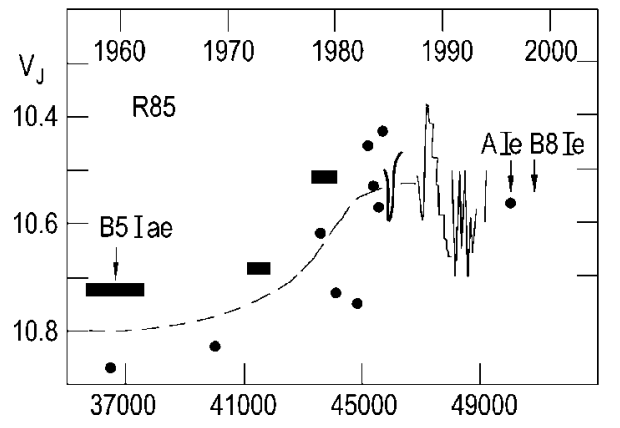

Fig. 13. The schematic light curve $\left(V_{\mathrm{J}}\right)$ versus JD-2400000 of the w-a S Dor variable R 85 in the LMC. Note that the magnitude HDEptg $1925=10.9$ (Table 5) is not indicated in the figure. The broken curve represents the L-SD phase. The S-SD phase and the $\alpha$ Cyg-type microvariations are schematically sketched on scale (based on $V B L U W$ monitoring, while the dots are in most cases individual observations by various authors), see for further details van Genderen et al. (1998b). The epochs of the three spectral type determinations are indicated by arrows and are from Feast et al. (1960), viz. around 1959, and Massey et al. (2000), viz. in 1996 and 1999 (Tables 4, 5 and 6)

the present paper). This group is divided into three subgroups, (1): the positive $(+),(2)$ : the negative $(-)$ candidates (the first ones show a stronger SD-signature than the second ones) and (3): the former, thus, noncandidates (0). It is now certain, or at least almost certain, that the last category do not belong to the $\mathrm{S}$ Dor variables. They all are listed in Tables 11, 12 and 13.

It must be stressed that the stars of the four categories a) to d) show almost without any exception microvariations as described in Sect. 1.6, at least if numerous photometric data are available. Tables 14 and 15 decode the reference numbers given in Tables $1-6$ and $8-13$ below the header "ref." and mentioned in the notes below the tables, where they are bracketed.

\subsection{The selection of parameters and their accuracy}

Photometric, spectroscopic and physical parameters for the same object often vary significantly from author to author. This diversity is caused by differences in assumptions and calibrations, apart from the variability of the objects. The temperature and luminosity determination of an object based on different methods may lead to substantial differences, even when applied by the same author.

Uncertainties arise for example in assigned spectral types and spectral analyses and consequently in the temperature. Optical and UV spectral types sometimes disagree. Wind asymmetries might be a plausible reason (Pasquali 1997). Temperatures derived from the intrinsic colour $(B-V)$ Jo may be unreliable due to a flatter energy distribution, the cause of the extended atmosphere. Additionally, interstellar reddening determinations are often uncertain. 


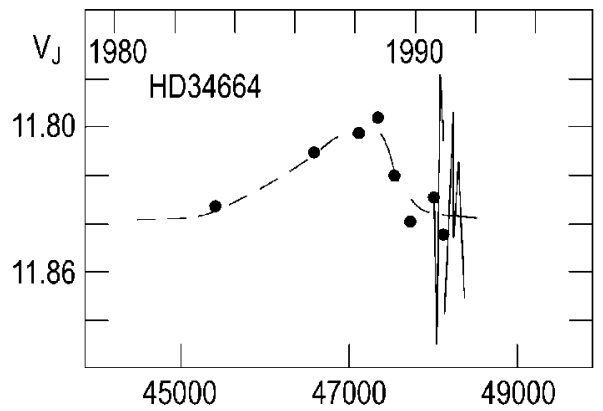

Fig. 16. The schematic light curve $\left(V_{\mathrm{J}}\right)$ versus JD-2440000) of the w-a S Dor $/ \mathrm{B}[\mathrm{e}]$ variable HD 34664 in the LMC. The dots are all averages of time series of $V B L U W$ photometry and clearly represent an S-SD phase since the colours become red in the maximum (van Genderen \& Sterken 1999). In order to illustrate the size of the $\alpha$ Cyg-type microvariations, a few of these time series are shown in detail, but only schematically sketched and on scale. Zickgraf et al. (1986) have given a compilation of scattered photometry, generally consisting of single observations, made between 1957 and 1984. These magnitudes $V_{\mathrm{J}}$ hover between 11.72 and 11.85 and are not shown

Further, the chosen apparent magnitudes of the extrema in the light curve, or the average apparent magnitude, to obtain $M_{V}$, as well as the applied $T_{\text {eff }} / \mathrm{BC}$ calibration to find $M_{\mathrm{bol}}$, may differ from author to author. And last but not least, the error in the distance can be considerable.

For galactic objects the errors in $\log T_{\text {eff }}$ can easily amount to up to $0.05(\sim 4000 \mathrm{~K}$ for high and $\sim 1000 \mathrm{~K}$ for low temperatures) and 0.2 in $\log L / L_{\odot}\left(0 .{ }^{\mathrm{m}} 5\right.$ in $\left.M_{\text {bol }}\right)$. For the Magellanic Cloud objects the errors in $\log T_{\text {eff }}$ will be the same as above, but relative errors in $\log L / L_{\odot}$ are much smaller than 0.2 , say about 0.03 for the LMC and 0.06 for the SMC objects (the difference between the two stellar systems is caused by the difference in inclination angle).

The errors in the temperature mentioned above introduce errors via the $\mathrm{BC}$ in $\log L / L_{\odot}$, thus $\pm 0.1 \mathrm{in} \log L / L_{\odot}$ for the hottest $\mathrm{S}$ Dor variables, while it is negligible for the cool ones. This error should be incorporated into the error in the luminosity (for the hottest variables) given above.

Apart from that, relative errors in the distance moduli between galactic and Magellanic Clouds amounting to $\sim 0$. 15 cause an extra relative error in $\log L / L_{\odot}$ of $\sim 0.06$.

In incidental cases the uncertainty in the distance of galactic objects is relatively larger, e.g. HD 80077, Cyg OB2\#12, WRA 751, He 3-519 and the Pistol Star. The error in $\log L / L_{\odot}$ may then amount to $0.4\left(1^{\mathrm{m}}\right.$ in $\left.M_{\mathrm{bol}}\right)$. For $\eta$ Car, the $M_{V}$ and the circumstellar reddening of the hidden S Dor variable are the main uncertain parameters, affecting its error box on the HR-diagram.

Because of these problems, I list parameters which often are an average of various sources. Only the (hopefully) most reliable parameters were selected. References on which these data are partly or completely based, are given also. To get a more or less homogeneous set, many values from the literature, if necessary, were slightly revised by applying the relation $A_{V}=3.1 E(B-V)$ and taking $M_{V \odot}=4.75$. If no temperatures were known, the spectral type, or an average of literature spectra, served as a temperature indicator by applying the calibrations of de Jager \& Nieuwenhuijzen (1987). The BC scale of Schmidt-Kaler (1982) was then used to derive $\mathrm{M}_{\mathrm{bol}}$. If no spectrum was known, $(B-V)$ Jo served as an temperature indicator.

Further, all Magellanic Cloud objects have been scaled to the same distance with the distance moduli 18.45 and 19.0 for LMC and SMC, respectively. This procedure may lead in some cases to smaller scatter, yet I will use the estimated errors given above for error bars on the HR-diagram (Sect. 3).

\section{The distribution of the $S$ Dor variables on the HR-diagram}

\subsection{The HR-diagram}

Figure 20 shows the position of the four categories of objects on the HR-diagram in four separate panels. The error bars are based on estimates (Sect. 2.2). The bars at the left represent the relative errors for the objects within the same stellar system. There are two bars at the right labeled "extra". The first one with the sublabel "dist" is due to the error in the distances of the three stellar systems relative to each other. The second one with the sublabel "BC hot" is the effect of an error in temperature on the luminosity via the BC. It is only appropriate for the hottest $\mathrm{S}$ Dor variables since the $\mathrm{BC}$ is negligible for the cooler ones. Both errors should be incorporated to the three at the left by adding them quadratically and taking the square root, if objects of one stellar system are compared with those in another one.

The dotted vertical line in each panel is the calculated threshold for dynamical instability when going from low to high temperatures according to Stothers \& Chin (1996). Indeed, only a few objects lie on the left of it and within the estimated error.

\subsection{Discussion on the HR-diagram}

\subsubsection{The s-a S Dor variables}

The upper left panel of Fig. 20 shows the s-a variables: 4 galactic, 2 SMC and 8 LMC objects (Tables 1, 2 and 3). To prevent crowding, not all designations are given. The minimum state (dots) and the maximum state (circles) are connected by a line. Most of these lines, representing schematically the displacement during SD-phases, run more or less horizontal as expected. The more luminous the star, the larger the shift in temperature and the visual amplitude. For HDE 269216 (LMC) only the average position is shown (open square), since no precise data for its extrema are known. For HDE 269582 (LMC) only the minimum position could be derived (dot). 


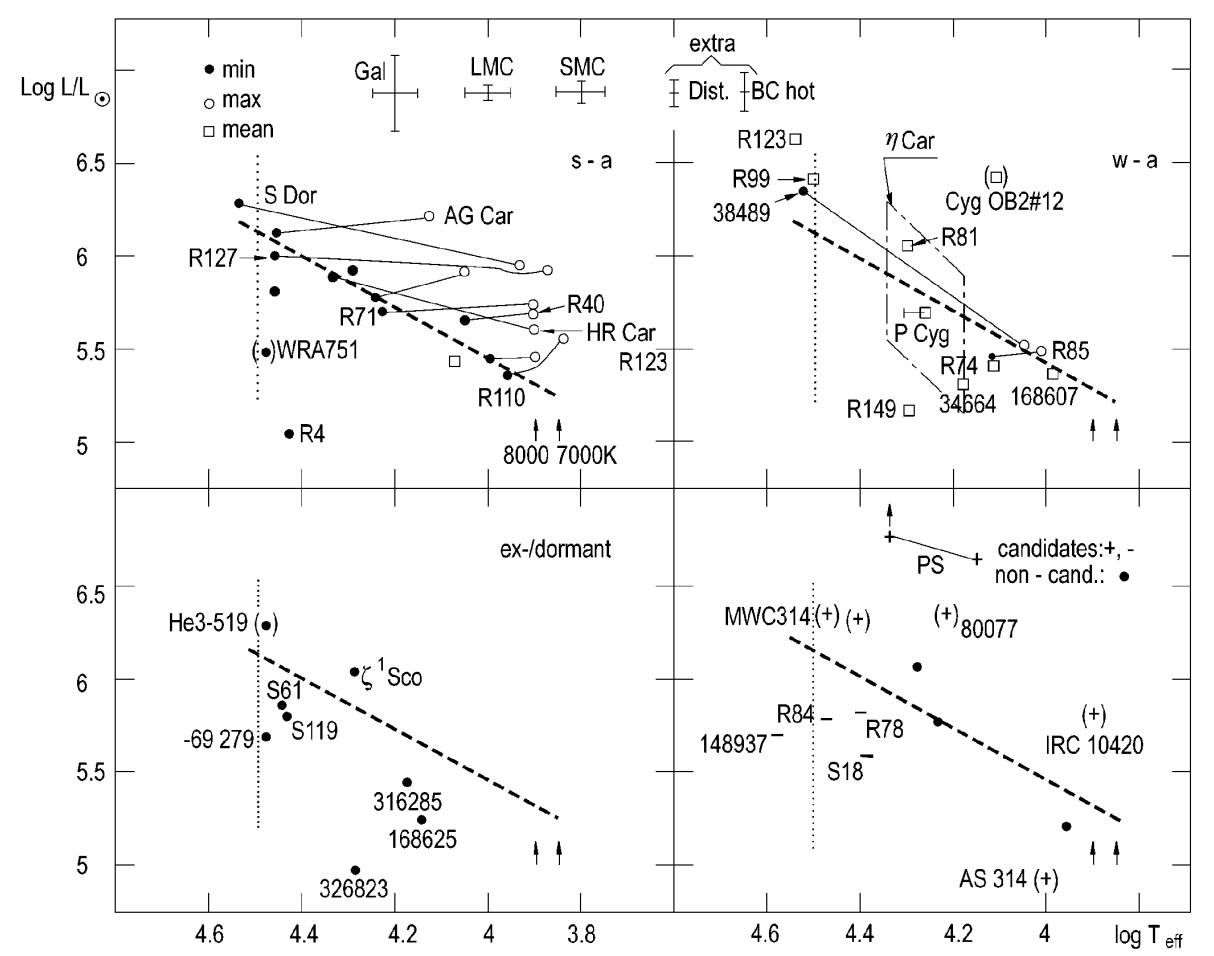

Fig. 20. The position of the four categories of S Dor variables on the theoretical HR-diagram. The fat dashed line in the four panels represents the SD-minimum strip. See for further explanation Sect. 3.2

The thick dashed line sketched through the minima (3 galactic, $1 \mathrm{SMC}$ and $7 \mathrm{LMC}$ objects) represents the "s-a SD-minimum strip", which will be called from now on the "SD-minimum strip". Such a relation has been first pointed out by Wolf (1989). The dashed line has been copied into the three other panels, which will be discussed hereafter. WRA 751 (uncertain distance) and R 4 (SMC; see below) have been ignored. The equation for the strip is:

$\log L / L_{\odot}=1.37 \log T_{\text {eff }}-0.03$.

It must be stressed that this strip is not necessarily the same as where the SD-eruptions originate. However, see the discussion on P Cyg in Sect. 3.2.2.

The small scatter is surprising. It can partly be explained by the fact that errors in temperature cause a change in luminosity via the $\mathrm{BC}$, which runs along almost the same inclination as the strip. If the small scatter is not accidental and intrinsic to the strip, then the distances are apparently rather reliable.

The SMC object R 4 (including an A-type companion) is much too faint: about 2.5 (bolometric) compared to other S Dor variables with the same temperature. This must be intrinsic. Whether this has anything to do with the fact that it also is a $\mathrm{B}[\mathrm{e}]$ star (Tables 1 and 2; see also van Genderen \& Sterken 1999) is unknown. B[e] stars are likely rapid rotators and according to Langer (1999) such stars should have a lower luminosity. On the other hand Langer \& Heger (1998) concluded from the morphology and chemical enrichment of the nebula that $\mathrm{R} 4$ was originally composed of a close binary and a third star (the
A-type companion). The close binary merged into a single star: the present $\mathrm{B}[\mathrm{e}] / \mathrm{S}$ Dor companion. In this model, supported by observations of the nebula by Pasquali et al. (2000), the merger star would be expected to add a large amount of He to the interior of the combined object, giving it a very large $L / M$ ratio. However, how should this be reconciled with the unusual low luminosity of $\mathrm{R} 4$ ?

The evolutionary tracks of stars on the verge of becoming a WR star leave the SD-area by bending down to the left (the most massive ones), or leaving it horizontally to the left (the less massive ones). Thus, $\mathrm{R} 4$ could be such a case.

The second SMC object in this group, R 40, lies only slightly above the SD-minimum strip. Clear systematic differences between the objects of the three stellar systems due to differences in metal content, are obviously absent. Perhaps R 40 is the only one that to some extent responds to the expectation, since with low $\mathrm{Z} L$ goes up and $T_{\text {eff }}$ drops (Stothers \& Chin 1996) and the location of the photospheric Eddington limit goes up (Lamers \& Noordhoek 1993). However, the observed deviation is not significant.

According to the opaque-wind model of Davidson (1987) the maximum visual brightness cannot surpass the temperature limit to the red between $7000 \mathrm{~K}$ and $8000 \mathrm{~K}$. Although an SD-phase is basically caused by an expanding radius and a decreasing temperature, rather than by an expanding pseudo-photosphere due to an increasing wind density, this group (and the other ones to be discussed below) seems to have a limit at $\sim 7000 \mathrm{~K}$. Perhaps the SD-phase is not only caused by a radius and 
temperature variation alone, but might at times be a mixture of an expanding radius/decreasing temperature and an expanding pseudo-photosphere, see van Genderen et al. (1998b, Sect. $\left.4.5^{1}\right)$

The SD-minimum strip lies roughly at the same location as that of the dynamical unstable blue models of Stothers \& Chin (1996), viz. between $\log L / L_{\odot} \sim 6.1$ and $\log T_{\text {eff }} \sim 4.5$, and $\log L / L_{\odot} \sim 5.4$ and $\log T_{\text {eff }} \sim 3.9$.

Further, it should be noted that the red side of the SD-area overlaps the "Yellow Void", a region of atmospheric instability of yellow hypergiants, investigated by Nieuwenhuijzen \& de Jager (1995, 2000) and de Jager (1998), see Sect. 3.2.5 of the present paper.

\subsubsection{The w-a S Dor variables}

The upper right panel shows the w-a members: 4 galactic and 8 LMC objects (Tables 4, 5 and 6 ). For most of these stars the parameters have been plotted as averages, since the extrema are not precisely known. HD 38489 is the only one with a relative large range: minimum (dot) and maximum (circle) are connected by a line, although the light variation is poorly known. R 85 has a small range, minimum (dot) and maximum (circle) are connected by a line. That the long-term low-amplitude variation represents an SD-phase indeed is obvious from the three spectral type determinations indicated in Fig. 13: at minimum the star is hotter than at the maximum.

Two famous objects: $\eta$ Car (Fig. 11) and P Cyg, belong to this group. The S Dor variable connected to the first object is hidden in a lot of circumstellar material, so its brightness and temperature are only roughly known (Table 4). According to the principle of a "Chinese lantern" any light variation of the variable is imitated by the bipolar reflection nebula (mainly by the dominant SE part more or less pointing to us, van Genderen et al. 1999). It appears that the light variation in the visual during the interval 1992-1994 showed strong similarities with that of R 85 during 1984-1986: both S Dor variables showed a cycle of an S-SD phase with microvariations superimposed (van Genderen et al. 1999). This proves that the S Dor variable hidden in $\eta$ Car must also be a normal $\mathrm{S}$ Dor variable. This is supported by the spectrum which looks like that of P Cyg (Ebbets et al. 1997). Its most probable position is outlined by the dashed-dotted error box. The cause of the large uncertainty is explained in the notes to Table 4. The present status of $\eta$ Car is discussed in Sect. 3.2.6.

\footnotetext{
1 Note that in this paper, we misinterpreted Stothers \& Chin's (1996) concept of the "optically thick ejected cloud" in point 4 of their conclusions. They expressed the view that the observable changes in the photometric characteristics are primarily produced by the optically thick cloud, as part of the expanding and contracting stellar envelope, if the latter does not become fully detached. According to them, the amount of mass loss may be even not much influenced during the instability cycles.
}

After the SD-eruptions in the 17th century, P Cyg's total displacement in $\log T_{\text {eff }}$ to the right on the HR-diagram amounts to 0.046, assuming constant luminosity (Lamers \& de Groot 1992; de Groot \& Lamers 1992). This is indicated by the short horizontal line in Fig. 20. If this is not much in error, the eruptions occurred close to its present position and according to the model of Langer et al. (1994), P Cyg ought to be at the beginning of an S Dor stage.

It is of interest to note that the age of the oldest shell of $\mathrm{P}$ Cyg, possibly a relic of an eruption, amounts to some $210^{4}$ yr (Meaburn et al. 1999). This suggests that $\mathrm{P}$ Cyg has been that long an S Dor variable. According to Stothers's (1999b) prediction, P Cyg is now exactly in a state of marginal dynamical instability, as is indeed observed by de Groot et al. (2001) (see Sect. 1.6 and Table 6 in the present paper). Only microvariations are present and a possible very low-amplitude SD-cycle.

The Stothers \& Chin (1995) models do not predict much variation in the luminosity, either during or directly after the mass-loss episodes. In the course of the "blue loop" after a mass-loss episode, the star, in this case $\mathrm{P}$ Cyg, should be (nearly) inactive, as is observed. Thus, the observations of $\mathrm{P}$ Cyg support my view that S Dor variables are a sub-class of the $\alpha$ Cyg variables (Sect. 1.6). It seems obvious that the microvariations are hardly influenced by the various SD-instabilities: they are present when the star is not, or at most an extremely weak-active $\mathrm{S}$ Dor variable, or they are imperturbably present during high-amplitude SD-phases. Presumably they should be considered as small-scale instabilities of the outer layers, typical for $\alpha$ Cyg variables in general.

If the $M_{\mathrm{bol}}$ of P Cyg was a magnitude brighter during the eruption of the 17 th century, which is not certain at all (Lamers \& de Groot 1992), the displacement followed an inclination steeper than the SD- minimum strip. The model of Langer et al. (1994) does not predict such a large variation in the luminosity.

With a few exceptions, the w-a members obey the SDminimum strip, but with more scatter. Most of them are Be stars and/or are suspected of having a gaseous disk e.g. R 99 and R 123 in the LMC (Table 4).

$\mathrm{R} 149$ (Be+neb, LMC) is like R 4 (B[e], SMC) in the previous panel, much too faint, which could be intrinsic (Sect. 3.2.1). I suspect that most of the w-a variables should fit the SD-minimum strip because they are supposed to be in a minimum of both types of SD-phases. The s-a variable HR Car experienced such a minimum lasting about $20 \mathrm{yr}$ and the s-a variable $\mathrm{R} 71$ has now (1999) been in such a state for $15 \mathrm{yr}$, only showing weak-active SD phases (Fig. 4; van Genderen et al. 1997b; Sterken et al. 1997b). Such minima might even last much longer. Note that R 81 (B2.5eq Ia ${ }^{+}, \mathrm{LMC}$ ) and R 123 (Bpec, LMC), were s-a variables in the previous century (Figs. 12 and 15 , respectively). 


\subsubsection{The ex-/dormant S Dor variables}

The bottom panel at the left of Fig. 20, shows the ex-/dormant variables: 5 galactic and 3 LMC objects (Tables 8, 9 and 10). Most of them lie below the SDminimum strip. Whether this is accidental or intrinsic (e.g. due to further evolution to the blue, see Sect. 3.2.1) is unknown. Again, there is one object with a much too low luminosity, the galactic object HDE 326823. Lopes et al. (1992) and Sterken et al. (1995b) suspect that it is on its way to the WN stage. One could speculate that the same is true for R 4 and R 149 discussed in Sects. 3.2.1 and 3.2.2, respectively.

According to Weis (1999) S 119 has a shell typical for an $\mathrm{S}$ Dor variable, although the age may amount up to $\sim 10^{5} \mathrm{yr}$, which is relatively high.

\subsubsection{The candidate and former candidate S Dor variables}

The bottom panel at the right of Fig. 20 shows the candidate $\mathrm{S}$ Dor variables, at least those for which luminosity and temperature are known: 6 positive candidates $(+\operatorname{sign}$, bracketed if the position is very uncertain) and 4 negative candidates (- sign) (Tables 11, 12 and 13). The Pistol Star (PS) is represented by two models. Their positions far from the SD-minimum strip could point to a large uncertainty in the distance and/or the reddening, just like for most of the other candidates. Further, 3 non-candidates (dots) are also plotted.

The positive candidate IRC +10420 is the only object which was situated on the left side of the $7000-8000 \mathrm{~K}$ border (discussed in Sect. 3.2.1) some decades ago, but which evolved quickly to about $7900 \mathrm{~K}$ in 1994 (Oudmaijer 1995; Nieuwenhuijzen \& de Jager 2000). The star has been plotted in Fig. 20 with the last-mentioned temperature. This object might still be a yellow hypergiant. See for a further discussion on the possible relationship between the two types of variables Sect. 3.2.5.

\subsubsection{Discussion on the possible evolutionary connection between S Dor variables and yellow hypergiants}

According to de Jager (1998) IRC +10420 is a yellow hypergiant, like $\rho$ Cas and HR 8752. HR 8752 appeared to have evolved quickly to the blue during the 20th century (Zsoldos 1986), like IRC +10420 (e.g. Kastner et al. 1995; Oudmaijer 1995; Nieuwenhuijzen \& de Jager 2000). $\rho$ Cas may be doing the same, although its behaviour is more chaotic.

The observations and calculations of Nieuwenhuijzen $\&$ de Jager $(1995,2000)$ and de Jager (1998) indicate that HR 8752 and $\rho$ Cas are now bouncing against the cool border $(\sim 7500 \mathrm{~K})$ of the Yellow Void, a region of atmospheric instability, which overlaps the cool side of the SDarea (although no S Dor variables reside in the Void, just at its lower boundary, see Fig. 21). This is accompanied by enhanced mass ejections. For HR 8752 the bouncing occurs in possible cycles of $\sim 10 \mathrm{yr}$. It is unknown how and when these objects will cross this border to enter the Yellow Void. With other words: are these fast evolving yellow hypergiants in fact proto-S Dor variables? The opposite question has been posed by de Jager (1998: Sect. 2). According to Garcia-Segura et al.'s (1996b) evolutionary model for a $35 M_{\odot}$ star: possibly not. Their model predicts an extremely rapid evolution from the yellow hypergiant stage to the WR stage: about $100 \mathrm{yr}$ !

However, as de Jager \& Nieuwenhuijzen (see above) have demonstrated, the evolution of the yellow hypergiants to the blue will be stopped by the Yellow Void for some time, but it is unknown for how long. Once they have passed, or avoided this barrier somehow, there is perhaps not much time left for a (blue) S Dor stage. On the other hand, note that the $\mathrm{S}$ Dor variables like the $\mathrm{w}$-a variables HD 168607, R 85, R 74, the s-a variable R 110 and the ex-/dormant variable HD 168625 in Fig. 20, lie just at the lower edge of the Yellow Void (Fig. 21) and that not one S Dor variable is situated right inside the Void, (only the s-a variables cross the Void during SD-phases and are obviously not in a normal state then).

Therefore, we may assume that once the envelopes of the yellow hypergiants have lost so much mass that it results in more atmospheric stability, they become lowluminous S Dor variables after all and for a time long enough to be observed. Note that both types of variables are generally blueward evolving stars and that the masses of the yellow hypergiants (Nieuwenhuijzen \& de Jager 2000) are most likely of the same order as that of the S Dor variables like R 110: $10 M_{\odot}$ (Stahl et al. 1990), HD 160529: $13 M_{\odot}$ (Sterken et al. 1991) and HD 168625: $10 M_{\odot}$ (Robberto \& Herbst 1998). Consequently, in some respects, yellow hypergiants could be very well protoS Dor variables, consistent with de Jager's (1998) supposition. Besides, the cycles of mass ejections of the yellow hypergiants and the SD-phases of S Dor variables may be related instability mechanisms.

It should be noted that yellow hypergiants also belong to the $\alpha \mathrm{Cyg}$ variables, viz. the cool ones, showing microvariations on a time scale of hundreds of days.

\subsubsection{The present status of $\eta \mathrm{Car}$}

The fact that all S Dor variables with reasonable reliable distances and reddenings lie on a relatively narrow band on the HR-diagram, can have consequences for an object like $\eta$ Car. Its distance $(2.3 \mathrm{kpc})$, foreground reddening $(0.50)$ and total luminosity $\left(\log L / L_{\odot}=6.65\right.$, or $M_{\text {bol }}=$ -11.9 ) seem to be reliable as well. Nevertheless, its position if plotted in Fig. 20 is too far above the S Dor area, whatever temperature is used. Note that more than $90 \%$ of the luminosity is due to IR radiation. Thus, apart from the S Dor variable, more IR sources, or sources leading to heating of dust and gas complexes (such like explosive events in the past, accreting mass processes, etc.), might 


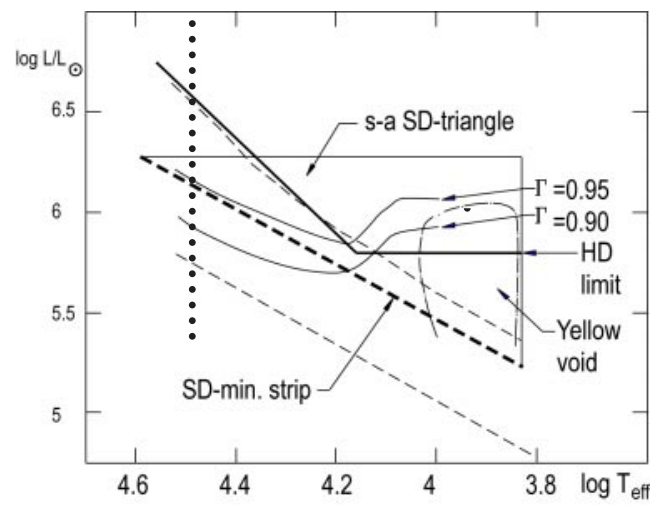

Fig. 21. The structure of the S Dor area on the HR-diagram. It shows amongst others the SD-triangle in which the s-a $\mathrm{S}$ Dor variables move to-and-fro during the SD-phases, the SDminimum strip (fat dashed line) with the thinly dashed lines as its bandwidth, and the Yellow Void

be present inside $\eta$ Car (van Genderen et al. 1994, 19952, 1999, 2001).

Note that the radial velocity curve based on lines of the Paschen series is useless and that according to Davidson et al. (2000) the binary hypothesis is weak, in contrast with the view of Damineli et al. (2000). The justification of serious doubt on the radial velocity curve of Damineli et al. (1997) is certainly not new: two years before the study of Davidson et al. (2000), de Groot \& Henderson (1998, quoted by van Genderen et al. 1999) already expressed their serious concern. They found that the HeI $5875 \AA$ and $6678 \AA$ lines (presumably originating deeper in the wind) gave radial velocities completely different from those on which the excisting binary models were based.

The "spectroscopic events", the X-ray observations and the spectroscopy suggest a colliding-wind binary (Damineli et al. 1997, 2000; Pittard 2000). Another indication that $\eta$ Car hides a secret is based on the detection of peculiar light variations of non-stellar origin, sometimes only clearly present in the near-UV, sometimes also detectable in $V$ and $B$ and deviating from the normal SD-variability. Most striking are the periodicities in the near-UV, detected by our multi-photometric monitoring campaigns. They could point to the presence of a luminous accretion disk which can occur in semi-detached binaries (van Genderen et al. 1994, 1995, 1999, 2001). Calculations based on formulae derived from energy balance arguments (Bath 1979; Bath \& Pringle 1985) show that variations in such a disk can be measurable. This would imply the presence of a semi-detached binary, but other scenarios without a semi-detached binary are not impossible as well (van Genderen et al. in prep.).

Our photometric analysis revealed that the nett rising trend of the brightness from the near-IR (see also

\footnotetext{
${ }^{2}$ In the latter paper the cause of the anti-correlation between the $\mathrm{H} \beta$ index and the continuum light should probably sought in the S Dor star and not in the excistence of an extended HII region. When the S Dor star becomes fainter by the microvariations, the $\mathrm{H} \beta$ emission becomes obviously stronger.
}

Whitelock et al. 1994) to the near-UV between 1974 and 1992 is not due to a star's photosphere, but due to variations in the amount of circumstellar hot gas and dust (van Genderen et al. 1994). This is supported by a nearIR study of Smith \& Gehrz (2000). They conclude that the near-IR variations are mainly due to morphological changes of the ejecta in the core and their variable illumination. In this respect it is of interest to mention that according to Viotti \& Rossi (1999) the core is likely enshrouded by a $1^{\prime \prime}-2^{\prime \prime}$ dense circumstellar cloud. They believe also that in the immediate surroundings of the core, dust is at present condensating from the stellar wind at a very high rate.

See for a bipolar nebula older than the Homunculus, Sect. 1.8.

\subsection{Discussion on the structure of the $S D$-area}

Figure 21 summarizes the structure of the SD-area. It shows the HD-limit, the triangle in which the s-a variables move to and fro during the SD-phases, the SD-minimum strip with its limits (thinly dashed lines) in which most of the S Dor variables reside, and the Yellow Void for yellow hypergiants.

The SD-area between the thinly dashed lines is 0.6 wide in $\log L / L_{\odot}$, or 1.55 in $M_{\text {bol }}$ and confined to the right of the instability boundary of Stothers \& Chin (1996) (vertical dotted line).

The close similarity between the slopes of the SDarea and the oblique part of the empirical HD-limit indicates that the SD-phenomenon is somehow related to the Eddington limit as expected (the locus where $g_{\text {eff }}$ $=0$, a situation favourable for instability and increasing mass loss, e.g. Lamers \& Noordhoek 1993; de Koter 1993; Humphreys \& Davidson 1994). The SD-upper limit slopes down more deeply to the red than the HD-limit.

Figure 21 also shows the atmospheric Eddington limits (AEL) for post-mainsequence stars (PMS) for constant Eddington factors $\Gamma=0.90$ and $0.95(Z=0.02)$. Those for $Z=0.008$ are not very different (Lamers 1997). Between and above these lines, the atmospheres are expected to become losely bound, thus, for $\Gamma \geqq 0.9$. These lines fit the upper part of the SD-minimum strip very well (contrary to the lower part). Lamers (1997) speculated that the objects with low luminosity do not come directly from the MS, but should be post-RSG, as opposed to those belonging to the upper part. According to the chemical abundances of the ejecta, with the exception of that of P Cyg, all could be post-RSG (Sect. 1.7). The calculations of Stothers \& Chin (1996) predict that most S Dor variables are in their second (thus hot/blue) SD-instability stage, thus, postRSG, supporting many observational findings. Thus, the reason why the high luminous variables do fit the AEL for PMS stars, while they are possibly post-RSG, is not clear. 


\subsection{The maximum light amplitude due to the SD-phases}

In Fig. 22, the relation between the observed maximum light amplitude $\left(\triangle V_{\max }\right)$, largely based on the schematic light curves presented here, see also Table 16 to be discussed in Sect. 3.5) and the luminosity at minimum apparent brightness $\left(\log L / L_{\odot} \min \right)$ is investigated. Note that such a relation has already been investigated by Wolf (1989) and called the "amplitude-luminosity" relation. The figure shows 12 s-a- (dots), $3 \mathrm{w}-\mathrm{a}-$ (circles) and 1 ex-/dormant (square) S Dor variables. Since most of the observed light amplitudes are lower limits (unless the objects were observed for a century or so, in which case the amplitude can be considered as statistically reliable upper limits), the arrows point to the right. Objects with unreliable parameters have not been plotted.

The curve represents the maximum excursions in the s-a SD-triangle from the SD-minimum strip to the boundary at $\log T_{\text {eff }}=3.84$ at constant luminosity. Thus, from the maximum observed shift in temperature follows $\triangle \mathrm{BC}_{\max }$, which is therefore equal to $\triangle V_{\max }$. The higher the luminosity, the larger the expected light amplitude (which agrees more or less with the observations), since the radiation pressure is an important agent of the SD-instability.

Most of the objects lie close to the curve. Part of the scatter must be caused by the fact that $\triangle V_{\max }$ is not always equal to $\triangle \mathrm{BC}_{\max }$ (thus, no precise horizontal displacement in the HR-diagram). Another part is due to errors in the physical parameters and the fact that the genuine maximum amplitude has not been observed.

Evidently, this maximum amplitude due to the sum of the two SD-phases (S-SD and L-SD phases) can amount to about 2.5 . Note that this is only slightly less than that for the SD-eruptions of $\eta$ Car and P Cyg!

\subsection{The duration of maximum and minimum state}

Variables currently in or close to minimum state, especially the w-a and ex-/dormant variables, may be on the verge of ending their S Dor-life, or may have just ended it. However, the probability that this is so, is low, as may be concluded from the following discussion. This seems to indicate that their relative quiescence is in most cases only temporary.

Table 16 lists the variables, ordered according to category, of which the probable apparent brightness before 1950 could be traced. The header brightness/activity is divided into two columns: $<1950$ and 1950-2000, listing whether the objects were in a "high" or "low" state. The column "ampl" lists the observed total light range of the SD-phases, which inevitably is in most cases a lower limit.

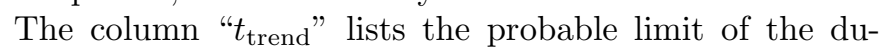
ration between "high" and "low" brightness of the SD-

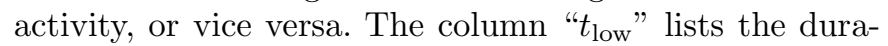
tion of the "low" state. The last column lists the reference numbers, or refers to a figure, or tables, in the present paper.

It appears that at least 8 objects: viz. $5 \mathrm{~s}-\mathrm{a}-, 2 \mathrm{w}-\mathrm{a}-$ and 1 ex-/dormant objects (uncertain cases are bracketed and are not included in this statistic), were presumably "high" before, in some cases long before, 1950. At least 4 objects: viz. 2 s-a-, $1 \mathrm{w}$-a- and 1 ex-/dormant objects were presumably "low" before, or long before, 1950. Of two specimens of the latter group, R 40 and R 127, together with R 110 ("low" state before 1950 uncertain) we witnessed their developing SD-phases in the last few decades. This means that the precise durations of the "high" and "low" states before 1950 are unknown because of the lack of observations.

It appears that most of the time scales of the declines and rises $\left(t_{\text {trend }}\right)$ lie between 20 and $50 \mathrm{yr}$. The durations of the observed non-active stages $\left(t_{\text {low }}\right)$ are of the same order. $\zeta^{1}$ Sco has already been low for $100 \mathrm{yr}$ (Sterken et al. 1997a). For HD 160529 the "high" state $\left(t_{\text {high not }}\right.$ listed in Table 16) already has lasted $110 \mathrm{yr}$ (Fig. 2). To this sample P Cyg can be added, which seems to have been hardly active for perhaps $300 \mathrm{yr}$ (however, a very low amplitude oscillation with a time scale of $8 \mathrm{yr}$ has been detected by de Groot et al. 2001; this could be an S-SD cycle, see Table 6).

Most of the objects have not been monitored photometrically for longer than a few decades (since 1950), thus, it is quite thinkable that most of the current (19502000) "low" or "nearly low" states are only temporary. It is therefore impossible to say which of the ex-/dormant variables are really "ex" and on their way to become a WN-type star.

If the numbers of variables are counted showing a low activity in the last two-three decades, say with light amplitudes $\lesssim 0$. 2 , (thus, difficult to discover), one finds about 24 (5 s-a-, $10 \mathrm{w}-\mathrm{a}-, 8 \mathrm{ex}-/$ dormant and 1 positive candidate, viz. HD 80077). The total number of variables amount to 35 (excluding the other 7 positive candidates, because there is no information on any light variation: most of them are still shrouded by dense circumstellar material).

On the other hand, there are only a few which turned out to have been highly, or moderately active for $110 \mathrm{yr}$ or more, viz. AG Car (van Genderen et al. 1997a), HR Car (but with a long lasting inactive interval, van Genderen et al. 1997b) and HD 160529 (see above), while S Dor (not listed in Table 16) has been active for at least $165 \mathrm{yr}$ (to be more precise since at least $\sim 1834$, see Thackeray 1974 ; van Genderen et al. 1997a). Some of the S Dor variables in M 31 and M 33 also show an activity lasting at least $100 \mathrm{yr}$ (AF And in M 31, Var.A in M 33), others are only active at intervals of $20 \mathrm{yr}$ (e.g. Var.C in M 33) in between periods of quiescence lasting a few decades (Hubble \& Sandage 1953; Sharov 1975, 1990; Rosino \& Bianchini 1973; Humphreys et al. 1988).

Consequently, the numbers and examples above suggest that $\mathrm{S}$ Dor variables are for most of their lifetime $(\sim 70 \%)$ close to $(=\mathrm{w}-\mathrm{a})$, or in a minimum (= dormant) state. Long-lasting active stages (thus, with frequent 


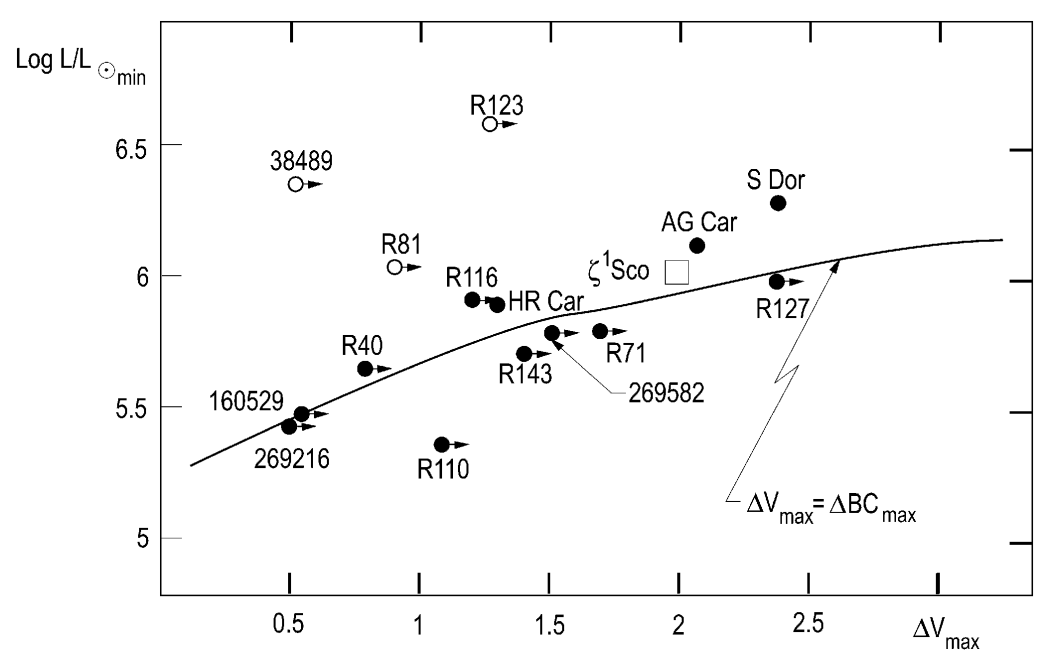

Fig. 22. The maximum observed light amplitude as a function of the luminosity at minimum apparent brightness. The relation between maximum light amplitudes in the s-a SD-triangle for genuine horizontal displacements on the HR-diagram (thus, then $\left.\triangle V_{\max }=\triangle B C_{\max }\right)$ is represented by the curved line

SD-phases) are rare. Whether S Dor variables can be very active for most of their lifetime is unknown, but obviously such objects are rare too.

\subsection{Total numbers of S Dor variables in the Galaxy and the Magellanic Clouds}

If the numbers of the three categories (s-a, w-a and ex-/dormant) are complete for the volume of the galactic disc around the Sun (maximum radii $6 \mathrm{kpc}$ for the s-aand $2.5 \mathrm{kpc}$ for the other two groups, excluding He3-519 with a possible distance of $8 \mathrm{kpc}$, see Table 9 ), then the total numbers in the galactic disk can be estimated. Table 17 summarizes the results. For the determination of the multiplication factors ("mult.f.") the radius of the galactic disk has been taken to be $12 \mathrm{kpc}$ with a negligible thickness. There should then be about $200 \mathrm{~S}$ Dor variables. If the number for the LMC is complete, which is not certain at all, there are 10 times more S Dor variables in the Galaxy than in the LMC. However, considering that the mass ratio between galactic disk and the LMC is presumably of the same order (80 and $1010^{9} M_{\odot}$, respectively), there is not much difference in their frequency.

Assuming that the incompleteness is the same for both Magellanic Clouds, the ratio found for the numbers of $\mathrm{S}$ Dor variables as well as for the masses of the two stellar systems one finds LMC/SMC $\sim 6$ (the mass for the SMC amounts to $1.510^{9} M_{\odot}$ ). Due to the low number of variables in the SMC, this result is not very reliable.

\section{Conclusions}

It was my intention to give a concise overview of the current knowledge on S Dor variables up to the beginning of the 21st century, complemented with an inventory of all confirmed and suspected members and members in quiescence. I also felt the need to put much emphasis on the photometric achievements of the various types of instabilities, because usually not enough light is shed thereon and besides a number of misunderstandings, continuously wandering through literature, needed clarification and correction.

S Dor variables exhibit a number of instabilities which, together with their precise evolutionary state, are highly of interest, but badly understood. None are strictly periodic like Cepheids and the like. The instabilities comprise: 1. the rare SD-eruptions (Sect. 1.4). Is stochastic resonance a cause, as tentatively suggested by Sterken et al. (1997a), possibly in connection with the dynamical ionization-induced dynamical instability discovered by Stothers \& Chin (1996)?

2. the frequent SD-phases (Sect. 1.5) consisting of two types with different time scales $(t<10 \mathrm{yr}$ and $t \gtrsim 20 \mathrm{yr}$, the short (S)-SD- and long (L)-SD phases, respectively) which are often superimposed on each other (Sect. 1.5.1). Is there any connection between these two and the SDeruptions? Where and how do they originate and how should the multi-periodic character of the SD-phases of e.g. AG Car be explained (Sects. 1.5.2 and 1.5.3)? Presumably, the SD-phases, the "secular cycles" according to Stothers \& Chin (1995, see Sect. 1.5.1 of the present paper) originate deeper in the stellar interior than the microvariations, otherwise the latters should not remain so imperturbably present on the ongoing SD-phases;

3 . the two types of microvariations (Sect. 1.6) with different timescales (weeks: near minimum light, and about 100 d: near maximum light). Within a certain temperature interval (20000-10000 K) they can be seen superimposed on each other. This suggests that they originate in different layers. Subsequently, e.g. when the SD-phase is on its way to maximum light, the short one quickly disappears (within a few months or shorter), the longer one continues. This must be due to changing physical conditions of the outer layers by the expansion. 
Thus, it appears that S Dor variables form a wellequipped laboratory for a number of photospheric and atmospheric pulsations and instabilities, but theoretically they are still badly understood. To cite Stothers (2000, priv. comm.): "A great difficulty right now seems to be the problem of correlating what the stellar models predict with what the observations show. This will continue to be a problem until theoretical models can be produced that are more sophisticated". Therefore, the modeling should continue, with the emphasis on the explanation for the photometric phenomena and to answer the question: will all $\alpha$ Cyg variables pass through a blue S Dor-stage?

Topics as well are the origin, the chemical and dynamical characteristics of their circumstellar ejecta which are usually much better understood. It appears that the chemistry and the dynamics of the ejecta are important for the interpretation of the evolutionary stage of S Dor variables: in most cases they point to a post-RSG stage, even for the most luminous variables.

The inventory is complemented with physical and photometric parameters (made as homogeneous as possible) and other characteristics for all members and suspected members, collected from literature. Attention was given to errors in temperature and luminosity, of crucial importance for the interpretation of their position on the theoretical HR-diagram.

The inventory contains 46 SDor variables among which 12 candidates and additionally it contains 3 rejected candidates. It is differentiated into four categories according to the size of the maximum light amplitude of the SD-phases exhibited in the 20th century. It appears that the strong-active S Dor variables obey the relation, called the SD-minimum strip:

$\log L / L_{\odot}=1.37 \log T_{\text {eff }}-0.03$.

The other categories obey the same relation, but with more scatter which is twice as large on the blue side than on the red side. This could point to evolutionary effects, or high rotation velocities. The occasional specimens even show a substantial and significant deviation from the narrow S Dor area (R 4 in the SMC, R 149 and HDE 326823 in the LMC). Thus, according to the observations, the S Dor stage occupies only a small portion of the blue part of the evolutionary tracks of massive stars.

In Sect. 3.2.5 a short discussion is given of the possible evolutionary connection between S Dor variables and the variable yellow hypergiants. These may be proto-lowluminous S Dor variables.

In Sect. 3.2.6 the present status of $\eta$ Car is discussed (see also Sect. 1.8). It has been confirmed that the reliability of the radial velocity curve on which the existing binary models were based, is highly questionable.

It would be a misunderstanding to believe that all S Dor variables are, or should be, spectacular during most of their lifetime. The majority appears to be lowamplitude $(<0.5)$ variables, or are close to the minimum state. It is estimated that for more than $70 \%$ of their lifetime as a hot S Dor variable, they are not spectacular at all. It was the happy few, like $\eta$ Car, P Cyg, S Dor, AG Car, etc., which gave the S Dor variables their famous reputation.

The estimated total number of S Dor variables in the Galaxy amounts to $\sim 200$. It seems that the frequency of $\mathrm{S}$ Dor variables in the Galaxy, the LMC and the SMC is more or less equal, although the frequency in the SMC is based on low-number statistic.

All reseachers interested in these variables should look eagerly forwards to surprises which the unpredictable S Dor variables have instore for us in the 21st century.

Note added in proof: It is quite well possible that $\mathrm{P}$ Cyg is yet slightly instable in view of the possible very low-amplitude SD-phase with a time scale of about $8 \mathrm{y}$ (Table 6). Very recent research by Markova and collaborators has demonstrated that for this variation a correlation excists between brightness, temperature and radius, similar to those of other S Dor variables during SD-phases. Further, it appeared that the massloss rate increased with increasing radius (like R 71 and S Dor) (Markova, N. 2000, ASP Conf. Ser., 204, 117; Markova, N. 2001, ASP Conf. Ser. on P Cyg, Armagh, in press; Markova, N., Morrison, N., Kolka, I., \& de Groot, M. 2001, in press; Markova, N., Scuderi, S., de Groot, M., \& Panagia, N. 2001, A\&A, in prep.).

This $8 \mathrm{y}$ variation and a $500 \mathrm{~d}$ variation are possibly cyclic, at least spectroscopically (Markova, N., Morrison, N., Kolka, I., \& de Groot, M. 2001, in prep.).

Further, it appeared that the $100 \mathrm{~d}$ microvariation of $\mathrm{P}$ Cyg (Table 6) has a counterpart in the wind variability (Markova, N., 2000, A\&AS, 144, 391; Markova, N., Scuderi, S., de Groot, M., \& Panagia, N. 2001, in prep).

The inner nebula within a few arcsec from HR Car (see Table 1) has been studied by Hulbert, S., Nota, A., Clampin, M., et al. 1999, IAU Coll., 169, 103, and White, S. M. 2000, ApJ, 539, 851.

R 143 (see Table 1) appears to have a clump of material which is definitely ejected by an SD-eruption according to Smith, L. J., Nota, A., Pasquali, A., et al. 1998, ApJ, 503, 278.

A dust model for the ejected nebula of G79.29+0.46 (see Table 11) has been presented by Trams, N. R., van Tuyll, C. I., Voors, R. H. M., et al. 1999, IAU Coll., 169,71 , and more proof of its membership as an S Dor variable has been offered by Voors, R. H. M., Geballe, T. R., Waters, L. B. F. M., et al. 2000, A\&A, 326, 236. Both references suggest for the central star $\log L / L_{\odot} \sim 5.48$ and $\log T_{\text {eff }} \sim 4.26$, thus, different from the values listed in Table 11.

A bipolar shell has been discovered recently around G25.5+0.2 (see Table 11) by Clark, J. S., Steele, I. A., \& Langer, N. 2000, ApJ, 541, L67.

Acknowledgements. I am much indebted to the following colleagues for their invaluable remarks and comments: Drs. R. B. Stothers, C. de Jager, C. Sterken, I. Salamanca and B. Wolf. 
I am also very grateful to Dr. J. K. Katgert-Merkelijn for improving the language.

\section{References}

Allen, D. A., \& Glass, I. 1976, ApJ, 210, 666

Allen, D. A., \& Hillier, D. J. 1993, Proc. Astron. Soc. Aust., 10,338

Appenzeller, I., \& Wolf, B. 1979, A\&AS, 38, 51

Appenzeller, I., Wolf, B., \& Stahl, O. 1987, in Instabilities in Luminous Early Type Stars, ed. H. J. G. L. M. Lamers, \& C. W. H. de Loore (Reidel), 241

Ardeberg, A., Brunet, J. P., Maurice, E., \& Prévot, L. 1972, A\&AS, 6, 249

Azzopardi, M., \& Vigneau, J. 1975, A\&AS, 22, 285

Barba, R. H., Niemela, V. S., Baume, G., \& Vasquez, R. A. 1995, ApJ, 446, L23

Barba, R. H., Niemela, V. S., \& Morel, N. 1997, ASP Conf. Ser., 120, 238, ed. A. Nota, \& H. J. G. L. M Lamers

Barlow, M. J., Drew, J. E., Meaburn, J., \& Massey, R. M. 1994, MNRAS, 268, L29

Bateson, F. M., \& Jones, A. F. 1994, Publ. Var. Star Sect. RASNZ, 19

Bath, G. T. 1979, Nature, 282, 274

Bath, G. T., \& Pringle, J. E. 1985, in Interacting Binary Stars, ed. J. E. Pringle, \& R. A. Wade (Cambridge Univ. Press), 177

Bieging, J. H., Abbott, D. C., \& Churchwell, E. B. 1989, ApJ, 340,518

Blöcker, T., Balega, Y., Hofmann, K. H., et al. 1999, A\&A, 348,805

Bohannan, B. 1997, ASP Conf. Ser., 120, 3, ed. A. Nota, \& H. J. G. L. M. Lamers

Bohannan, B., \& Walborn, N. R. 1989, PASP, 101, 520

Bohigas, J., Tapia, M., Ruiz, M. T., \& Roth, M. 2000, MNRAS, 312, 295

Breysacher, J. 1997, ASP Conf. Ser., 120, 227, ed. A. Nota, \& H. J. G. L. M. Lamers

Breysacher, J., \& Perrier, C. 1991, IAU Coll., 143, 229, ed. K. A. van der Hucht, \& B. Hidayat

Brunet, J. P. 1975, A\&A, 43, 345

Burgarella, D., \& Paresce, F. 1991, A\&A, 241, 595

Burki, G., Heck, A., Bianchi, L., \& Cassatella, A. 1982, A\&A, 107,205

Carpay, J., de Jager, C., Nieuwenhuijzen, H., \& Moffat, A. F. J. 1989, A\&A, 216, 143

Carpay, J., de Jager, C., \& Nieuwenhuijzen, H. 1991, A\&A, 248,475

Cassinelli, J. P. 1999, ASP Conf. Ser., 179, 258, ed. J. A. Morse, R. M. Humphreys, \& A. Damineli

Catchpole, R. M., Whitelock, P. A., \& Glass, I. 1990, MNRAS, 247,479

Chentsov, E. L., \& Luud, L. 1990, Astrophys., 31, 415

Chini, R., Elsasser, H., \& Neckel, Th. 1980, A\&A, 91, 186

Clampin, M., Schulte-Ladbeck, R. E., Nota, A., et al. 1995, AJ, 110, 251

Clayton, G. C., \& Martin, P. G. 1985, ApJ, 288, 558

Code, A. D., \& Houck, T. E. 1958, PASP, 70, 261

Conti, P. S., Garmany, C. D., \& Massey, P. 1986, AJ, 92, 48

Corcoran, M. F., Swank, J. H., Petre, R., et al. 1999, ASP Conf. Ser., 179, 46, ed. J. A. Morse, R. M. Humphreys, \& A. Damineli
Cotera, A. S., Erickson, E. F., Colgan, S. W. J., et al. 1996, ApJ, 461, 750

Cox, A. N., Guzik, J. A., \& Soukup, M. S. 1995, ASP Conf. Ser., 83, 192, ed. R. S. Stobie, \& P. A. Whitelock

Cox, A. N., Guzik, J. A., \& Soukup, M. S. 1997, ASP Conf. Ser., 120, 133, ed. A. Nota, \& H. J. G. L. M. Lamers

Cox, P., Mezger, P. G., Sievers, A., et al. 1995, A\&A, 297, 168

Crowther, P. A., Hillier, D. J., \& Smith, L. J. 1995, A\&A, 293, 172

Crowther, P. A., \& Smith, L. J. 1997, A\&A, 320, 500

Damineli, A. 1996, ApJ, 460, L49

Damineli, A., Conti, P. S., \& Lopes, D. F. 1997, New Astron., 2,107

Damineli, A., Kaufer, A., Wolf, B., et al. 2000, ApJ, 528, L101

Davidson, K. 1987, ApJ, 317, 760

Davidson, K. 1989, IAU Coll., 113, ed. K. Davidson, A. F. J. Moffat, \& H. J. G. L. M. Lamers

Davidson, K., \& Humphreys, R. M. 1997, ARA\&A, 35, 1

Davidson, K., Ebbets, D., Johansson, S., et al. 1997, AJ, 113, 335

Davidson, K., Humphreys, R. M., Hajian, A., \& Terzian, Y. 1993, ApJ, 411, 336

Davidson, K., Gull, T. R., Humphreys, R. M., et al. 1999, AJ, 118,1777

Davidson, K., Ishibashi, K., Gull, T. R., et al. 2000, ApJ, 530, L107

Dong, Y. S., \& Hu, J. Y. 1991, Chin A\&A, 15, 275

Dufour, R. J., \& Duval, J. E. 1975, PASP, 87, 769

Duncan, R. A., White, S. M., Reynolds, J. E., \& Lim, J. 1999, ASP Conf. Ser., 179, 54, ed. J. A. Morse, R. M. Humphreys, \& A. Damineli

Duschl, W. J., Hofmann, K. H., Rigaut, F., \& Weigelt, G., 1995, Rev. Mex. AA SDc, 2, 41

Dwarkadas, V. V., \& Balick, B. 1998, AJ, 116, 829

Ebbets, D., Morse, J., Davidson, K., \& Walborn, N. R. 1997, ApJ, 489, L161

Feast, M. W., Thackeray, A. D., \& Wesselink, A. J. 1960, MNRAS, 121, 337

Figer, D. F., Najarro, F., Morris, M., et al. 1998, ApJ, 506, 384

Friedjung, M., \& Muratorio, G. 1980, A\&A, 85, 233

Gäng, T. 2000, Thesis Univ. of Heidelberg

Garcia-Lario, P., Riera, A., \& Manchado, A. 1998, A\&A, 334, 1007

Garcia-Segura, G., Mac Low, M. M., \& Langer, N. 1996a, A\&A, 305, 229

Garcia-Segura, G., Langer, N., \& Mac Low, M. M. 1996b, A\&A, 316, 133

Gautschy, A., \& Saio, H. 1995, ARA\&A, 33, 75

Gautschy, A., \& Saio, H. 1996, ARA\&A, 34, 551

van Genderen, A. M. 1970, A\&A, 7, 49

van Genderen, A. M. 1979a, A\&AS, 38, 249

van Genderen, A. M. 1979b, A\&AS, 38, 381

van Genderen, A. M. 1989, A\&A, 208, 135

van Genderen, A. M. 1991a, in ESO Workshop on Rapid Variability of OB Stars: Nature and Diagnostic Value, ed. D. Baade, 117

van Genderen, A. M. 1991b, IAU Symp., 143, 563, ed. K. A. van der Hucht, \& B. Hidayat

van Genderen, A. M. 2000, J. Astron. Data, 4

van Genderen, A. M., \& Sterken, C. 1996, A\&A, 308, 763

van Genderen, A. M., \& Sterken, C. 1999, A\&A, 349, 537

van Genderen, A. M., \& Sterken, C. 2001, in preparation

van Genderen, A. M., \& Thé, P. S. 1984, Sp. Sc. Rev., 39, 317 
van Genderen, A. M., van Leeuwen, F., \& Brand, J. 1982, A\&AS, 47, 591

van Genderen, A. M., Groot, M., \& Thé, P. S., 1983, A\&A, 117,53

van Genderen, A. M., Bijleveld, W., \& van Groningen, E. 1984, A\&AS, 58,537

van Genderen, A. M., Steemers, W. J. G., Feldbrugge, P. T. M., et al. 1985, A\&A, 153, 163

van Genderen, A. M., Thé, P. S., Augusteijn, Th., et al. 1988, A\&AS, 74, 453

van Genderen, A. M., Bovenschen, H., Engelsman, E. C., et al. 1989, A\&AS, 79, 263

van Genderen, A. M., Thé, P. S., Heemskerk, M., et al. 1990, A\&AS, 82, 189

van Genderen, A. M., Robijn, F. H. A., van Esch, B. P. M., \& Lamers, H. J. G. L. M. 1991, A\&A, 246, 407

van Genderen, A. M., Thé, P. S., de Winter, D., et al. 1992a, A\&A, 258, 316

van Genderen, A. M., van den Bosch, F. C., Dessing, F., et al. 1992b, A\&A, 264, 88

van Genderen, A. M., de Groot, M., \& Thé, P. S. 1994, A\&A, 283,89

van Genderen, A. M., Sterken, C., de Groot, M., et al. 1995, A\&A, 304, 415

van Genderen, A. M., Sterken, C., \& de Groot, M. 1997a, A\&A, 318,81

van Genderen, A. M., de Groot, M., \& Sterken, C. 1997b, A\&AS, 124,517

van Genderen, A. M., Sterken, C., de Groot, M., \& Reijns, R. A. 1998a, A\&A, 332, 857

van Genderen, A. M., Sterken, C., \& de Groot, M. 1998b, A\&A, 337, 393

van Genderen, A. M., Sterken, C., de Groot, M., \& Burki, G. 1999, A\&A, 343, 847

van Genderen, A. M., de Groot, M., \& Sterken, C. 2001, ASP Conf. Ser., in press

van Gent, R. H., \& Lamers, H. J. G. L. M. 1986, A\&A, 158, 335

Glatzel, W. 1997, ASP Conf. Ser., 120, 128, ed. A. Nota, \& H. J. G. L. M. Lamers

Glatzel, W., \& Kiriakidis, M. 1993, MNRAS, 263, 375

Glover, T. W., Dufour, R. J., Hester, J. J., et al. 1997, ASP Conf. Ser., 120, 296, ed. A. Nota, \& H. J. G. L. M. Lamers Gottlieb, E. W., \& Liller, Wm. 1978, ApJ, 225, 488

de Groot, M. 1969, BAN, 20, 225

de Groot, M., \& Lamers, H. J. G. L. M. 1992, Nature, 355, 422

de Groot, M., van Genderen, A. M., \& Sterken, C. 1996, Irish Astron. J., 23, 187

de Groot, M., van Genderen, A. M., \& Sterken, C. 1997a, Astron. Astrophys. Trans., 14, 35

de Groot, M., van Genderen, A. M., \& Sterken, C. 1997b, AAVSO Conf. Proc. (May 1997, Switserland)

de Groot, M., Sterken, C., \& van Genderen, A. M. 2001, in preparation

Guzik, J. A., Cox, A. N., Despain, K. M., \& Soukup, M. S. 1997, ASP Conf. Ser., 120, 138, ed. A. Nota, \& H. J. G. L. M. Lamers

Guzik, J. A., Cox., A. N., Despain, K. M., \& Soukup, M. S. 1999, IAU Coll., 169, 337, ed. B. Wolf, O. Stahl, \& A. W. Fullerton

Hamann, F., De Poy, D. L., Johansson, S., \& Elias, J. 1994, ApJ, 422, 626

Henize, K. G. 1952, ApJ, 115, 133
Henize, K. G. 1976, ApJS, 30, 491

Heydari-Malayeri, M., Rauw, G., Esslinger, O., \& Beuzit, J. L. 1997a, A\&A, 322, 554

Heydari-Malayeri, M., Courbin, F., Rauw, G., et al. 1997b, A\&A, 326, 143

Higgs, L. A., Wendker, H. J., \& Landecker, T. L. 1994, A\&A, 291, 295

Hillier, D. J., \& Allen, D. A. 1992, A\&A, 262, 153

Hillier, D. J., Crowther, P. A., Najarro, F., \& Fullerton, A. W. 1998, A\&A, 340, 483

Hiltner, W. A. 1954, ApJ, 120, 41

Hiltner, W. A. 1956, ApJS, 2, 389

Hoekzema, N., Lamers, H. J. G. L. M., \& van Genderen, A. M. 1992, A\&A, 257, 118

Hoekzema, N., Lamers, H. J. G. L. M., \& van Genderen, A. M. 1993, A\&AS, 98, 505

Hoffleit, D. 1933, Harv. Bull., 892, 19

Hoffleit, D. 1940, Harv. Bull., 913, 4

Hu, J. Y., de Winter, D., Thé P. S., \& Pérez, M. R. 1990, A\&A, 227, L17

Hubble, E., \& Sandage, A. 1953, ApJ, 118, 353

Humphreys, R. M. 1978, ApJS, 38, 309

Humphreys, R. M. 1999, IAU Coll., 169, 243, ed. B. Wolf, O. Stahl, \& A. W. Fullerton

Humphreys, R. M., \& Davidson, K. 1994, PASP, 106, 1025

Humphreys, R. M., Davidson, K., \& Smith, N. 2000, PASP, 111,1124

Humphreys, R. M., Lamers, H. J. G. L. M., Hoekzema, N., \& Cassatella, A. 1989, A\&A, 218, L17

Humphreys, R. M., Leitherer, C., Stahl, O., et al. 1988, A\&A, 203, 306

Hutsemékers, D. 1997, ASP Conf. Ser., 120, 316, ed. A. Nota, \& H. J. G. L. M. Lamers

Hutsemékers, D., \& van Drom, E. 1991, A\&A, 248, 141

Hutsemékers, D., van Drom, E., Gosset, E., \& Melnick, J. 1994, A\&A, 290, 900

Iben Jr., I. 1999, ASP Conf. Ser., 179, 367, ed. J. A. Morse, R. M. Humphreys, \& A. Damineli

Icke, V. 1981, ApJ, 247, 152

Innes, R. T. A. 1903, Cape Ann., 9, 75B

Israelian, G., \& de Groot, M. 1999, Sp. Sc. Rev., 90, 493

Isserstedt, J. 1975, A\&AS, 19, 259

de Jager, C. 1998, A\&A Rev., 8, 145

de Jager, C., \& van Genderen, A. M. 1989, IAU Coll., 113, 127 , ed. K. Davidson, H. J. G. L. M. Lamers, \& A. F. J. Moffat de Jager, C., \& Nieuwenhuijzen, H. 1987, A\&A, 177, 217

Jones, A., \& Sterken, C. 1997, J. Astron. Data, 3, 1

Jones, C. A., Chetin, T., \& Liller, W. 1974, ApJ, 190, L1

Jones, T. J., Humphreys, R. M., Gehrz, R. D., et al. 1993, ApJ, 411,323

Kaper, L., Lamers, H. J. G. L. M., Raymakers, E., et al. 1995, A\&A, 300, 446

Kaplan, S. A., \& Pikelner, S. B. 1970, The Interstellar Medium, Cambridge, Harvard Univ. Press, 50

Kastner, J. H., \& Weintraub, D. A. 1995, ApJ, 452, 833

Kiriakidis, M., Chernigovski, S., Fricke, K. J., \& Glatzel, W. 1997, ASP Conf. Ser., 120, 150, ed. A. Nota, \& H. J. G. L. M. Lamers

Kiriakidis, M., Fricke, K. J., \& Glatzel, W. 1993, MNRAS, 264, 50

Klochkova, V. G., Chentsov, E. L., \& Panchuk, V. E. 1997, MNRAS, 292, 19

Knoechel, G., \& Moffat, A. F. J. 1982, A\&A, 110, 263 
Koenigsberger, G., Auer, L. H., Georgiev, L., \& Guinan, E. 1998a, ApJ, 496, 934

Koenigsberger, G., Pena, M., \& Schmutz, W. 1998b, ApJ, 499, 889

Kolka, I. 1983, Esti NSV Tead. Akad. Toim. Fuus. Matem., 32, 51

Kolka, I. 1999, IAU Coll., 169, 268, ed. B. Wolf, O. Stahl, \& A. W. Fullerton

de Koter, A. 1993, Thesis Univ. Utrecht

de Koter, A., Scholten, G. G. J., \& Lamers, H. J. G. L. M. 1993a, Chap. 5 in Thesis de Koter (1993)

de Koter, A., \& Lamers, H. J. G. L. M. 1993b, Chap. 7 in Thesis de Koter (1993)

de Koter, A., Lamers, H. J. G. L. M., \& Schmutz, W. 1996, A\&A, 306, 501

Kozak, J. R. 1985, A\&AS, 62, 7

Kukarkin, B. V., Kholopov, P. N., \& Efremov, Yu. N. 1974, Sec. Suppl. Third Edition Gen. Cat. Var. Stars, Nauka, Moscow, 10

Lamers, H. J. G. L. M. 1987, in Instabilities in Luminous EarlyType Stars, ed. H. J. G. L. M. Lamers, \& C. W. H. de Loore (Reidel), 99

Lamers, H. J. G. L. M. 1995, ASP Conf. Ser., 83, 176, ed. R. S. Stobie, \& P. A. Whitelock

Lamers, H. J. G. L. M. 1997, ASP Conf. Ser., 120, 76, ed. A. Nota, \& H. J. G. L. M. Lamers

Lamers, H. J. G. L. M., \& de Groot, M. 1992, A\&A, 257, 153

Lamers, H. J. G. L. M., \& Noordhoek, R. 1993, ASP Conf. Ser., 35, 517, ed. J. Cassinelli, \& E. Churchwell

Lamers, H. J. G. L. M., de Groot, M., \& Cassatella, A. 1983, A\&A, 128, 299

Lamers, H. J. G. L. M., Hoekzema, N., Trams, N., et al. 1989, IAU Coll., 113, 271, ed. K. Davidson, A. F. J. Moffat, \& H. J. G. L. M. Lamers

Lamers, H. J. G. L. M., Bastiaanse, M. V., Aerts, C., \& Spoon, H. W. W. 1998, A\&A, 335, 605

Langer, N. 1999, IAU Coll., 169, 359, ed. B. Wolf, O. Stahl, \& A. W. Fullerton

Langer, N., Garcia-Segura, G., \& Mac Low, M. M. 1999, ApJ, 520, L49

Langer, N., Hamann, W. R., Lennon, M., et al. 1994, A\&A, 290,819

Langer, N., \& Heger, A. 1998, in B[e] Stars, ed. A. M. Hubert, \& C. Jaschek, ASSL 233, 235

van Leeuwen, F., van Genderen, A. M., \& Zegelaar, I. 1998, A\&AS, 127, 1

Leitherer, C. 1997, ASP Conf. Ser., 120, 213, ed. A. Nota, \& H. J. G. L. M. Lamers

Leitherer, C., \& Chavarria, K. C. 1987, A\&A, 175, 208

Leitherer, C., \& Wolf, B. 1984, A\&A, 132, 151

Leitherer, C., Allen, R., Altner, B., et al. 1994, ApJ, 428, 292

Leitherer, C., Appenzeller, I., Klare, G., et al. 1985, A\&A, 153, 168

Leitherer, C., Schmutz, W., Abbott, D. C., et al. 1989, ApJ, 346,919

Lennon, D. J., \& Dufour, P. L. 1986, A\&A, 155, 79

Lennon, D. J., Wobig, D., Kudritzki, R. P., \& Stahl, O. 1994, Sp. Sci. Rev., 66, 207

Lester, J. B., Gray, R. O., \& Kurucz, R. L. 1986, ApJS, 61, 509

Lopes, D. F., Damineli Neto, A., \& de Freitas Pacheco, J. A. 1992, A\&A, 261, 482

Lovey, D., Maeder, A., Noëls, A., \& Gabriël, M. 1984, A\&A, 133,307
Maeder, A. 1989, in Physics of Luminous Blue Variables, ed. K. Davidson, A. F. J. Moffat, \& H. J. G. L. M. Lamers (Kluwer), 15

Maeder, A. 1992, in Instabilities in Evolved Super- and Hypergiants, ed. C. de Jager, \& H. Nieuwenhuijzen (NorthHolland), 138

Maeder, A. 1997, ASP Conf. Ser., 120, 374, ed. A. Nota, \& H. J. G. L. M. Lamers

Maeder, A. 1999, IAU Symp., 193, 177, ed. K. A. van der Hucht, G. Koenigsberger, \& P. R. J. Eenens

Manfroid, J., Sterken, C., Bruch, A., et al. 1991, A\&AS, 87, 481

Manfroid, J., Sterken, C., Conuw, B., et al. 1994, A\&AS, 109, 329

Markova, N. 1986, A\&A, 162, L3

Markova, N., \& de Groot, M. 1997, A\&A, 326, 1111

Markova, N., \& Tomov, N. 1998, IBVS, 4641

Marston, A. P. 1999, IAU Symp., 193, 306, ed. K. A. van der Hucht, G. Koenigsberger, \& P. R. J. Eenens

Marston, A. P., Yocum, D. R., Garcia-Segura, G., \& Chu, Y. H. 1994, ApJ, 95, 151

Martini, A. 1969, A\&A, 3, 443

Massey, P. 2000, PASP, 112, 144

Massey, P., \& Thompson, A. B. 1991, AJ, 101, 1408

Massey, P., Waterhouse, E., \& Degioia-Eastwood, K. 2000, in press

McGregor, P. J., Hyland, A. R., \& Hillier, D. J. 1988a, ApJ, 324, 1071

McGregor, P. J., Finlayson, K., Hyland, A. R., et al. 1988b, ApJ, 329, 874

McGregor, P. J., Hyland, A. R., \& McGinn, M. T. 1989, A\&A, 223, 237

Meaburn, J., Walsh, J. R., \& Wolstencroft, R. D. 1993, A\&A, 268,283

Meaburn, J., Lopez, J. A., Barlow, M. J., \& Drew, J. E. 1996, MNRAS, 283, L69

Meaburn, J., Lopez, J. A., \& O'Connor, J. 1999, ApJ, 516, L29

Mendoza, V. E. E. 1970, Bol. Obs. Tonantzintla Tacubaja, 5, 269

Menshchikov, A. B. 1989, AN, 310, 335

Miroschnichenko, A. S. 1996, A\&A, 312, 941

Miroschnichenko, A. S., Chentsov, E. L., \& Klochkova, V. G. 1999, IAU Coll., 169, 272, ed. B. Wolf, O. Stahl, \& A. W. Fullerton

Moffat, A. F. J., \& FitzGerald, M. 1977, A\&A, 54, 263

Moffat, A. F. J., Marchenko, S. V., Bartzakos, P., et al. 1998, ApJ, 497, 896

Morris, P. W., Waters, L. B. F. M., Barlow, M. J., et al. 1999, Nature, 402, 502

Muratorio, G., \& Friedjung, G. 1988, A\&A, 190, 103

Najarro, F., Hillier, D. J., \& Stahl, O. 1997, A\&A, 326, 1117

Nieuwenhuijzen, H., \& de Jager, C. 1995, A\&A, 302, 811

Nieuwenhuijzen, H., \& de Jager, C. 2000, A\&A, 353, 163

Nota, A. 1999, IAU Coll., 169, 62, ed. B. Wolf, O. Stahl, \& A. W. Fullerton

Nota, A., \& Clampin, M. 1997, ASP Conf. Ser., 120, 303, ed. A. Nota, \& H. J. G. L. M. Lamers

Nota, A., Drissen, L., Clampin, M., et al. 1994, in Circumstellar Media in the Late Stages of Stellar Evolution, ed. R. Clegg, et al., Cambridge, 89

Nota, A., Livio, M., \& Schulte-Ladbeck, R. E. 1995a, ApJ, 448, 788

Nota, A., Pasquali, A., Drissen, L., et al. 1995b, ApJS, 102, 383 
Nota, A., Pasquali, A., Clampin, M., et al. 1996a, ApJ, 473, 946

Nota, A., Pasquali, A., Clampin, M., et al. 1996b, Proc. 33rd Liège Inst. Astr. Coll., Wolf-Rayet Stars in the Frame Work of Stellar Evolution, ed. J. M. Vreux, et al., 453

Nota, A., Smith, L., Pasquali, A., et al. 1997, ApJ, 486, 338

Ødegaard, K. J. R. 1999, IAU Coll., 169, 353, ed. B. Wolf, O. Stahl, \& A. W. Fullerton

Oudmaijer, R. D. 1995, Thesis, Univ. Groningen

Oudmaijer, R. D., 1998, A\&AS, 129, 541

Oudmaijer, R. D., Geballe, T. R., Waters, L. B. F. M., \& Sahu, K. C. 1994, A\&A, 281, L33

Oudmaijer, R. D., Groenewegen, M. A. T., Matthews, H. E., et al. 1996, MNRAS, 280, 1062

Owocki, S. P., \& Gayley, K. G. 1997, ASP Conf. Ser., 120, 121, ed. A. Nota, \& H. J. G. L. M. Lamers

Pacheco, J. A., Neto, A. D., Costa, R. D. D., \& Viotti, R. 1992, A\&A, 266, 360

Pamyathnykh, A. A. 1998, ASP Conf. Ser., 135, 268, ed. P. A. Bradley, \& J. A. Guzik

Pantin, E., \& Le Mignant, D. 2000, A\&A, 355, 155

Parker, J. W. 1997, ASP Conf. Ser., 120, 368, ed. A. Nota, \& H. J. G. L. M. Lamers

Parker, J. W., Clayton, G. C., Winge, C., et al. 1993, ApJ, 409, 770

Pasquali, A. 1997, ASP Conf. Ser., 120, 13, ed. A. Nota, \& H. J. G. L. M. Lamers

Pasquali, A., Langer, N., Schmutz, W., et al. 1997a, ApJ, 478, 340

Pasquali, A., Schmutz, W., Nota, A., \& Origlia, L. 1997b, A\&A, 327, 265

Pasquali, A., Nota, A., \& Clampin, M. 1999, A\&A, 343, 536

Pasquali, A., Nota, A., Langer, N., et al. 2000, AJ, 119, 1352

Pauldrach, A. W. A., \& Puls, J. 1990, A\&A, 237, 409

Percy, J. R., Napke, A. E., Richer, M. G., et al. 1988, A\&A, 191,248

Perry, C. L., Hill, G., \& Christodoulou, D. M. 1991, A\&AS, 90, 195

Pittard, J. M. 2000, Thesis, Univ. of Birmingham

Polomsk, E. F., Telesco, C. M., Piña, R. K., \& Fisher, R. S. 1999, AJ, 118, 2369

Pottasch, S. R., Wesselius, P. R., \& van Duinen, R. J. 1976, A\&A, 47, 443

Prinja, R. K., \& Schild, H. 1991, A\&A, 249, 428

Reddish, V. C., Lawrence, L. C., \& Pratt, M. N. 1967, Publ. R. Obs. Edinburgh, 5, 111

Rivinius, Th., Stahl, O., Wolf, B., et al. 1997, A\&A, 318, 819

Robberto, M., Ferrari, A., Nota, A., \& Paresce, F. 1993, A\&A, 269,330

Robberto, M., \& Herbst, T. M. 1998, ApJ, 498, 400

Rosino, L., \& Bianchini, A. 1973, A\&A, 22, 453

Schaller, G., Schaerer, D., Meynet, G., \& Maeder, A. 1992, A\&AS, 96, 269

Schild, H. 1987, A\&A, 173, 405

Schild, H., Hiltner, W. A., \& Sanduleak, N. 1969, ApJ, 156, 609

Schmidt-Kaler, Th. 1982, Landolt-Börnstein, Gruppe IV, Band 2

Schmutz, W. 1997, ASP Conf. Ser., 120, 115, ed. A. Nota, \& H. J. G. L. M. Lamers

Schmutz, W., Leitherer, C., Hubeny, I., et al. 1991, ApJ, 372, 664

Schulte, D. H. 1958, ApJ, 128, 41
Schulte-Ladbeck, R. E., \& Clayton, G. C. 1993, AJ, 106, 790 Schulte-Ladbeck, R. E., Clayton, G. C., Hillier, D. J., et al. 1994, ApJ, 429, 846

Sharov, A. S. 1973, IAU Symp., 67, 275, ed. V. E. Sherwood, \& L. Plaut

Shore, S. N. 1990, IAU Circ., 5009

Shore, S. N. 1992, in Nonisotropic and Variable Outflows from Stars, ed. L. Drissen, C. Leitherer, \& A. Nota, 342

Shore, S. N., \& Sanduleak, N. 1984, ApJS, 55, 1

Shore, S. N., Sanduleak, N., \& Allen, D. A. 1987, A\&A, 176, 59

Shore, S. N., Brown, D. N., Bopp, B. W., et al. 1990, ApJS, 73,461

Shore, S. N., Altner, B., \& Waxin, I. 1996, AJ, 112, 2744

Skinner, C. J. 1997, ASP Conf. Ser., 120, 322, ed. A. Nota, \& H. J. G. L. M. Lamers

Skinner, C., Becker, R. H., White, R. L., et al. 1998, MNRAS, 296,669

Smith, L. J. 1997, ASP Conf. Ser., 120, 310, ed. A. Nota, \& H. J. G. L. M. Lamers

Smith, L. J., Crowther, P. A., \& Prinja, R. K. 1994, A\&A, 281, 833

Smith, L. J., Stroud, M. P., Esteban, C., \& Vilchez, J. M. 1997, MNRAS, 290, 265

Smith, L. J., Nota, A., Pasquali, A., et al. 1998, ApJ, 503, 278

Smith, N., Gehrz, R. D., \& Krautter, J. 1998, AJ, 116, 1332

Smith, N., \& Gehrz, R. D. 2000, ApJ, 529, L99

Smith Neubig, M. M., \& Bruhweiler, F. C. 1999, AJ, 117, 2856

Soukup, M. S., Cox, A. N., Guzik, J. A., \& Morgan, S. M. 1994, BAAS, 26, 907

Souza, S. P., \& Lutz, B. L. 1980, ApJ, 235, L87

Spoon, H. W. W., de Koter, A., Sterken, C., et al. A\&AS, 106, 141

Stahl, O. 1986, A\&A, 164, 321

Stahl, O., \& Wolf, B. 1982, A\&A, 110, 272

Stahl, O., \& Wolf, B. 1986, A\&A, 154, 243

Stahl, O., \& Wolf, B. 1987, A\&A, 181, 293

Stahl, O., Wolf, B., Klare, G., et al. 1983, A\&A, 127, 49

Stahl, O., Leitherer, C., Wolf, B., \& Zickgraf, F. J. 1984a, A\&A, 131, L5

Stahl, O., Wolf, B., Leitherer, C., et al. 1984b, A\&A, 140, 459

Stahl, O., Wolf, B., de Groot, M., et al. 1985, A\&AS, 61, 237

Stahl, O., Wolf, B., \& Zickgraf, F. J. 1987, A\&A, 184, 193

Stahl, O., Wolf, B., Klare, G., et al. 1990, A\&A, 228, 379

Steemers, W. J. G., \& van Genderen, A. M. 1986, A\&A, 154, 308

Sterken, C. 1976, A\&A, 47, 453

Sterken, C. 1977, A\&A, 57, 361

Sterken, C. 1981, in The Most Massive Stars, ESO Workshop, ed. S. D'Odorico, D. Baade, \& K. Kjär, 147

Sterken, C., IAU Coll., 113, 59, ed. K. Davidson, A. F. J. Moffat, \& H. J. G. L. M. Lamers

Sterken, C., \& Breysacher, J. 1997, A\&A, 328, 269

Sterken, C., Gosset, E., Jüttner, A., et al. 1991, A\&A, 247, 383

Sterken, C., Manfroid, J., Anton, K., et al. 1993, A\&AS, 102, 79

Sterken, C., Manfroid, J., Beele, D., et al. 1995a, A\&AS, 113, 31

Sterken, C., Stahl, O., Wolf, B., et al. 1995b, A\&A, 303, 766

Sterken, C., Jones, A., Vos, B., et al. 1996a, IAU Circ., 4401

Sterken, C., de Groot, M., \& van Genderen, A. M. 1996b, A\&AS, 116, 9

Sterken, C., Jones, A., van Genderen, A. M., \& de Groot, M. 1996c, Australian J. Astr., 6, 199 
Sterken, C., de Groot, M., \& van Genderen, A. M. 1997a, A\&A, 326,640

Sterken, C., van Genderen, A. M., \& de Groot, M. 1997b, ASP Conf. Ser., 120, 35, ed. A. Nota, \& H. J. G. L. M. Lamers

Sterken, C., de Groot, M., \& van Genderen, A. M. 1998, A\&A, 333,565

Sterken, C., Freyhammer, L., Arentoft, T., \& van Genderen, A. M. 1999a, A\&A, 346, L33

Sterken, C., van Genderen, A. M., de Groot, M., \& Burki, G. 1999b, ASP Conf. Ser., 179, 200, ed. J. A. Morse, R. M. Humphreys, \& A. Damineli

Sterken, C., Arentoft, T., Duerbeck, H. W., \& Brogt, E. 1999c, A\&A, 349, 537

Sterken, C., et al. 2001, A\&A, in preparation

Stothers, R. B. 1999a, ApJ, 513, 460

Stothers, R. B. 1999b, ApJ, 516, 366

Stothers, R. B. 1999c, MNRAS, 305, 365

Stothers, R. B. 2000, ApJ, 530, L103

Stothers, R. B., \& Chin, C. W. 1993, ApJ, 408, L85

Stothers, R. B., \& Chin, C. W. 1994, ApJ, 426, L43

Stothers, R. B., \& Chin, C. W. 1995, ApJ, 451, L61

Stothers, R. B., \& Chin, C. W. 1996, ApJ, 468, 842

Stothers, R. B., \& Chin, C. W. 1997, ApJ, 489, 319

Stothers, R. B., \& Chin, C. W. 1999, ApJ, 522, 960

Stothers, R. B., \& Chin, C. W. 2000, ApJ, 540, 1041

Stroud, M. P. 1997, ASP Conf. Ser., 120, 350, ed. A. Nota, \& H. J. G. L. M. Lamers

Subrahmanyan, R., Ekers, R. D., Wilson, W. E., et al. 1993, MNRAS, 263, 868

Szeifert, Th., Stahl, O., Wolf, B., et al. 1993, A\&A, 280, 508

Thackeray, A. D. 1974, MNRAS, 168, 221

Thompson, G. I., Nandy, K., Morgan, D. H., et al. 1982, MNRAS, 200, 551

Torres-Dodgen, A. V., Tapia, M., \& Carroll M. 1991, MNRAS, 249, 1

Turner, D. G. 1985, A\&A, 144, 241

Vacca, W. D., \& Torres-Dodgen, A. V. 1990, ApJS, 73, 685

Vakili, F., Mourard, D., Stee, P., \& Bonneau, D. 1999, IAU Coll., 169, 87, ed. B. Wolf, O. Stahl, \& A. W. Fullerton

Vanbeveren, D., van Rensbergen, W., \& de Loore, C. 1998, The Brightest Binaries, Sect. 4 (Kluwer)

de Vaucouleurs, G., \& Eggen, D. J. 1952, PASP, 64, 185

van der Veen, W. E. C. J., Waters, L. B. F. M., Trams, N. R., \& Mathews, H. E. 1994, A\&A, 285, 551

Venn, K. 1997, ASP Conf. Ser., 120, 95, ed. A. Nota, \& H. J. G. L. M. Lamers

Viotti, R. 1971, PASP, 83, 170

Viotti, R., \& Rossi, C. 1999, ASP Conf. Ser., 179, 211, ed. J. A. Morse, R. M. Humphreys, \& A. Damineli

Viotti, R., Cassatella, A., Ponz, D., \& Thé, P. S. 1988, A\&A, 190, 333

Viotti, R., Rossi, L., Cassatella, A., et al. 1989, ApJS, 71, 983

Voors, R. H. M. 1999, Thesis Univ. Utrecht

Voors, R. H. M., Waters, L. B. F. M., Trams, N. R., \& Käufl, H. U. 1997, A\&A, 321, L21

Voors, R. H. M., Waters, L. B. F. M., Morris, P. W., et al. 1999, A\&A, 341, L67

Voors, R. H. M., Waters, L. B. F. M., de Koter, A., et al. 2000, Obs., in press

Waelkens, C., Aerts, C., Kestens, E., et al. 1998, A\&A, 330, 215

Walborn, N. R. 1982, ApJ, 256, 452

Walborn, N. R. 1989, IAU Coll., 113, 250, ed. K. Davidson, A. F. J. Moffat, \& H. J. G. L. M. Lamers
Walborn, N. R., \& Blades, J. C. 1997, ApJS, 112, 457

Walborn, N. R., Blanco, B. M., \& Thackeray, A. D. 1978, ApJ, 219, 498

Walborn, N. R., Evans, I. N., Fitzpatrick, E. L., et al. 1991, IAU Symp., 143, 505, ed. K. A. van der Hucht, \& B. Hidayat

Walraven, Th., \& Walraven, J. H. 1977, A\&AS, 30, 245

Walsh, J. R., \& Ageorges, N. 2000, A\&A, 357, 255

Warren, P. R., \& Penfold, J. E. 1975, MNRAS, 172, 41P

Waters, L. B. F. M., Izumiura, H., Zaal, P. A., et al. 1996, A\&A, 313, 866

Weis, K. 1996, Diplomarbeit, Ruprecht-Karls-Univ., Heidelberg

Weis, K. 1999, Inaugural-Dissertation, Ruprecht-Karls-Univ., Heidelberg, AA, in press

Weis, K. 2000, A\&A, 357, 938

Weis, K., Bomans, D. J., Chu, Y. H., et al. 1995, Rev. Mex. A. A. Ser. Conf., 3, 237

Weis, K., Duschl, W. J., Bomans, D. J., et al. 1997, A\&A, 320, 568

Weis, K., Duschl, W. J., \& Chu, Y. H. 1999, A\&A, 349, 467

Westerlund, B. E. 1961, Uppsala Astron. Obs. Ann., 5, 1

Whitelock, P. A., Feast, M. W., Carter, B. S., et al. 1983a, MNRAS, 203, 385

Whitelock, P. A., Carter, B. S., Roberts, G., et al. 1983b, MNRAS, 205, 577

Whitelock, P. A., Feast, M. W., Koen, C., et al. 1994, MNRAS, 270,364

de Winter, D. 1996, Thesis Univ. Amsterdam

de Winter, D., Pérez, M. R., Hu, J. Y., \& Thé, P. S. 1992, A\&A, 257, 632

Wisse, P. N. J., \& Wisse, M. 1971, A\&A, 12, 149

Wolf, B. 1989, A\&A, 217, 87

Wolf, B., Stahl, O., de Groot, M., \& Sterken, C. 1981, A\&A, 99,351

Wray, J. 1966, Ph.D. Thesis, North Western Univ.

Zickgraf, F. J. 1997, ASP Conf. Ser., 120, 173, ed. A. Nota, \& H. J. G. L. M. Lamers

Zickgraf, F. J., Wolf, B., Stahl, O., et al. 1985, A\&A, 143, 421

Zickgraf, F. J., Wolf, B., Stahl, O., et al. 1986, A\&A, 163, 119

Zickgraf, F. J., \& Schulte-Ladbeck, R. E. 1989a, A\&A, 214, 274

Zickgraf, F. J., Wolf, B., Stahl, O., \& Humphreys, R. M. 1989b, A\&A, 220, 206

Zickgraf, F. J., Kovács, J., Wolf, B., et al. 1996a, A\&A, 309, 505

Zickgraf, F. J., Humphreys, R. M., Lamers, H. J. G. L. M., et al. 1996b, A\&A, 315, 510

Zsoldos, E. 1986, Obs., 106, 156

\section{The inventory}

\subsection{Format of the tables of the inventory}

All sources indicated by reference numbers, in the tables below the header "ref", are codified in Tables 14 and 15. Uncertain data in the tables have been bracketed. Below the tables special notes are given with bracketed reference numbers. 


\subsubsection{The s-a S Dor variables in Tables 1,2 and 3}

\section{Table 1}

Column 1: Most popular designations by a name and/or a catalogue number, for 4 galactic-, 2 SMC- and 8 LMC s-a S Dor variables, respectively.

Column 2: Approximate dates of the extrema for which the physical and photometric parameters in this table and Table 2 are applicable.

Columns 3, 4 and 5: Effective temperature, absolute visual magnitude and luminosity, respectively. Blanks: no parameters could be derived with any certainty.

Column 6: Reference numbers on which the parameters in Cols. 3-5 are partly, or completely based; pp $=$ present paper.

Column 7: Gas nebula (g), or dust nebula (d) present? $\mathrm{y}=$ yes, $\mathrm{n}=\mathrm{no}, ?=$ still uncertain, blank $=$ unknown.

Column 8: Reference numbers concerning Col. 7, discussing and/or summarizing physical, chemical and geometrical parameters and giving references.

\section{Table 2}

Column 1: A selected designation (see Table 1).

Columns 2 and 3: Reddening (the subscript J refers to the $U B V$ system of Johnson) and reference numbers respectively.

Columns 4 and 5: The distance in kpc for the galactic objects and reference numbers, respectively. $\mathrm{pp}=$ present paper. See for the distance moduli of the SMC and LMC Sect. 2.2.

Columns 6, 7 and 8: The apparent visual magnitude $V_{\mathrm{J}}$ (the subscript $\mathrm{J}$ refers to the $V$ of the $U B V$ system of Johnson) of the extrema (dates listed in Table 1) and the reference numbers, respectively.

Column 9: A selected list of additional reference numbers dealing with photometry, spectroscopy and /or on various aspects of possible SD-signatures. Remarks on early magnitude determinations are given in square brackets, see for more (44). They are important for the determination of a possible long-term light variation. However, an uncertainty of a few tenths of a magnitude should be taken into account.

\section{Table 3}

Column 1: A selected designation (see Table 1).

Column 2: Approximate time scale (first line) and amplitude (second line) of the L-SD phases.

Column 3: The same as in Col. 2, but now for the S-SD phases.

Column 4: The same as in Cols. 2 and 3, but now for the microvariations.

Column 5: Reference numbers concerning Cols. 2, 3 and 4.

Column 6: A reference to figures with a schematic light curve and to Table 16 with some information on a possible long-term light variation.

\subsubsection{The w-a S Dor variables in Tables 4,5 and 6}

\section{Table 4}

Column 1: Most popular designations by a name and/or a catalogue number, for 4 galactic- and 8 LMC w-a S Dor variables, respectively.

Column 2: A selected spectral classification.

Column 3: Approximate date(s), or approximate time interval, for which the physical and photometric parameters in this table and Table 5 are applicable.

Columns 4, 5 and 6: The effective temperature, the absolute visual magnitude (the apparent visual magnitude used is listed in Table 5 in italics) and the luminosity, respectively.

Column \%: Reference numbers on which the parameters in Cols. 4, 5 and 6 are partly, or completely based. pp = present paper.

Columns 8 and 9: The same as Cols. 7 and 8 in Table 1.

\section{Table 5}

Column 1: A selected designation (see Table 4).

Columns 2, 3, 4 and 5: The same as in Table 2.

Columns 6, 7 and 8: The mean (Col. 6) and/or minimum apparent visual magnitude (Col. 7) and reference numbers, respectively. The magnitudes in italics have been used for $M_{V}$ and $\log L / L_{\odot}$ in Table 4 . The maximum apparent magnitude can be easily derived from the difference between the minimum and the mean magnitudes.

Column 9: The same as in Table 2.

\section{Table 6}

Column 1: A selected designation (see Table 4).

Columns 2, 3, 4, 5 and 6: The same as in Table 3.

\subsubsection{The ex-/dormant $\mathrm{S}$ Dor variables in Tables 8,9 and 10}

\section{Table 8}

Column 1: Most popular designations by a name, or by a catalogue number, for 5 galactic- and 3 LMC ex-/dormant S Dor variables, respectively.

Column 2: A selected spectral classification.

Column 3: Approximate time interval for which the physical and photometric parameters in this table and Table 9 are applicable.

Columns 4, 5 and 6: The effective temperature, the absolute visual magnitude (the mean apparent visual magnitude used is listed in Table 9) and the luminosity, respectively.

Column \%: Reference numbers on which the parameters in Cols. 4, 5 and 6 are partly or completely based. pp = present paper.

Columns 8 and 9: The same as Cols. 7 and 8, respectively, in Table 1.

\section{Table 9}

Column 1: A selected designation (see Table 8).

Columns 2, 3, 4 and 5: The same as in Table 2. 
Columns 6 and \%: The mean apparent visual magnitude and reference numbers, respectively.

Column 8: The same as Col. 9 in Table 2.

Table 10

Column 1: A selected designation (see Table 8).

Column 2, 3, 4, 5 and 6: The same as in Table 3.

\subsubsection{The candidate and former candidate $\mathrm{S}$ Dor variables in Tables 11, 12 and 13}

\section{Table 11}

Column 1: Most popular designation by a name and/or a catalogue number for 8 galactic-, 2 SMC- and 2 LMC candidate $\mathrm{S}$ Dor variables and 3 former candidates (marked by a "0": 1 star in the Galaxy and 2 stars in the LMC).

Column 2: A selected spectral classification.

Column 3: The weight for the candidate membership: "+" = indications for a possible S Dor-membership; "-" $=$ indications are weaker than for the previous category; "0" = most likely no S Dor variable, but it is a normal $\alpha$ Cyg variable.
Column 4: Approximate date, or time interval, for which the photometric and physical parameters in this table and in Table 12 are applicable.

Columns 5, 6, 7 and 8: The same as Cols. 4, 5, 6 and 7 , respectively, in Table 8.

Columns 9 and 10: The same as Cols. 7 and 8 in Table 1.

\section{Table 12}

Columns 1: A selected designation (see Table 11).

Columns 2, 3, 4 and 5: The same as in Table 2.

Columns 6 and \%: The mean apparent visual magnitude and reference numbers, respectively.

Column 8: The same as Col. 9 in Table 2.

\section{Table 13}

Column 1: A selected designation (see Table 11).

Column 2: The same as Col. 3 in Table 11.

Column 3: The approximate time scales, or quasiperiods (first line) and amplitudes (second line) of the microvariations.

Column 4: Reference numbers concerning Col. 3.

Column 5: A reference to figures with a schematic light curve. 